\title{
CHARACTERISTIC MATRICES AND SPECTRAL PROPERTIES OF EVOLUTIONARY SYSTEMS
}

\author{
M. A. KAASHOEK AND S. M. VERDUYN LUNEL
}

\begin{abstract}
In this paper we introduce the notion of a characteristic matrix for a large class of unbounded operators and study the precise connection between characteristic matrices and spectral properties of evolutionary systems. In particular, we study so-called multiplicity theorems. Several examples will illustrate our results.
\end{abstract}

\section{INTRODUCTION}

Many problems involving differential equations may be analyzed in terms of some holomorphic matrix function which in a natural way is associated with the problem. For example, to find the solutions of an ordinary differential equation

$$
\dot{x}=A x, \quad x(0)=x_{0},
$$

where $A$ is an $n \times n$-matrix and $x_{0} \in \mathbb{C}^{n}$, one has to analyze the matrix function

$$
\Delta(z)=z I-A \text {. }
$$

Indeed, if $m=m(\lambda, \Delta)$ denotes the multiplicity of $\lambda$ as zero of $\operatorname{det} \Delta$ and $k=k(\lambda, \Delta)$ denotes the multiplicity of $\lambda$ as pole of the matrix function $\Delta^{-1}$, then there exist $m$ independent solutions of the form $p(t) e^{\lambda t}$, where $\lambda$ is a zero of $\operatorname{det} \Delta$ and $p$ is a polynomial of degree at most $k-1$ whose coefficients may be described in terms of the Jordan chains of the matrix $\Delta$ at $\lambda$. In the language of matrices this observation is equivalent to

$$
\operatorname{dim} \operatorname{Ker}\left((\lambda I-A)^{k}\right)=m
$$

and follows from the Jordan canonical form for $A$.

There are important classes of infinite dimensional problems where a similar phenomenon appears. To illustrate this, consider the following retarded functional differential equation (RFDE) (see Hale [13] and Verduyn Lunel [30])

$$
\dot{x}(t)=L x_{t},
$$

where $L$ is a continuous mapping from the space of continuous functions $C[-h, 0]$ into $\mathbb{R}^{n}$ and $x_{t} \in C[-h, 0]$ denotes the state

$$
x_{t}(\theta)=x(t+\theta) \text { for }-h \leq \theta \leq 0 .
$$

Received by the editors August 30, 1990.

1991 Mathematics Subject Classification. Primary 34K05, 45E10. 
To find elementary solutions for (3), we substitute solutions of the form $x(t)=$ $e^{\lambda t} x_{0}$ into (3) and find that $\lambda$ must satisfy

$$
\operatorname{det} \Delta(\lambda)=0,
$$

where

$$
\Delta(z)=z I-L e_{z}
$$

and $e_{z} \in C[-h, 0]$ denotes the exponential function $e_{z}(\theta)=e^{z \theta}$. In general, equation (4) is an entire function of positive exponential type and has an infinite number of roots. But one can prove that there are exactly $m(\lambda, \Delta)$ independent elementary solutions of the form $p(t) e^{\lambda t}$, where $p$ is a polynomial of degree at most $k(\lambda, \Delta)-1$ and $\lambda$ a zero of $\operatorname{det} \Delta$. This result is known as "a folk theorem in functional differential equations" and was first proved by Levinger [22].

In a more abstract approach towards (3) the connection between (1) and (3) can be made even closer. Translation along the solution defines a strongly continuous semigroup $S(t): X \rightarrow X$ of bounded operators on a Banach space $X$ for both (1) and (3), that is,

(i) $S(0)=I$;

(ii) $S\left(t_{1}+t_{2}\right)=S\left(t_{1}\right) S\left(t_{2}\right)$ for $t_{1}, t_{2} \geq 0$;

(iii) $S(t) \varphi-\varphi \rightarrow 0$ in norm as $t \downarrow 0$.

For equation (1) the semigroup is given by $e^{A t}: \mathbb{C}^{n} \rightarrow \mathbb{C}^{n}$

$$
x_{0} \mapsto e^{A t} x_{0}
$$

and, in fact, is a group of bounded operators. For equation (3) the semigroup is given by $T(t): C[-h, 0] \rightarrow C[-h, 0]$

$$
\varphi \mapsto x_{t}(\cdot ; \varphi) \text {. }
$$

In this last case the one-parameter semigroup $T(t)$ does not extend to a group as can easily be seen from the property that ( 3 ) yields a bound for the derivative of the solution and hence, the solution becomes more smooth, i.e., differentiable on $[0, h]$, twice differentiable on $[h, 2 h]$, etc.

The infinitesimal generator $A(X \rightarrow X)$ for a strongly continuous semigroup $S(t)$ is defined by

$$
A \varphi=\lim _{t \downarrow 0} \frac{1}{t}[S(t) \varphi-\varphi],
$$

where the domain of $A$ equals the set of $\varphi$ such that the limit in (8) exists. The general theory, e.g., Pazy [27], implies that $A$ is a closed densely defined (unbounded) operator. For the semigroup given by (6) the infinitesimal generator is just the matrix $A$, but for the semigroup $T(t)$ given by (7) the infinitesimal generator is indeed unbounded:

$$
\mathscr{D}(A)=\{\varphi \in C[-h, 0]: \dot{\varphi} \in C[-h, 0], \dot{\varphi}(0)=L \varphi\}, \quad A \varphi=\dot{\varphi} .
$$

The elementary solutions of (3) are eigenfunctions and generalized eigenfunctions of $A$ and the spectrum of $A$ just contains point spectrum and is given by

$$
\sigma(A)=\{z: \operatorname{det} \Delta(z)=0\},
$$


where $\Delta$ is given by (5). Thus, the "folk theorem in functional differential equations" can be rephrased as a multiplicity theorem

$$
\operatorname{dim} \operatorname{Ker}\left((\lambda I-A)^{k(\lambda, \Delta)}\right)=m(\lambda, \Delta)
$$

which yields the same formula for the unbounded generator $A$ defined by (9) as we found before in the matrix case.

The fact that one can find a holomorphic matrix function $\Delta$ for an unbounded operator such that (11) holds is not uncommon. For example, the system of hyperbolic partial differential equations studied by Lopes et al. [23] also satisfies this property as was recently shown by Neves and Lin [26].

In this paper we describe in more explicit detail the connection between the operator $A$ and the matrix function $\Delta$, and we extend these results to other classes of equations. To be precise, we show that $A$ and $\Delta$ are related through an equivalence relation

$$
F(z)\left(\begin{array}{cc}
I & 0 \\
0 & z-A
\end{array}\right) E(z)=\left(\begin{array}{cc}
\Delta(z) & 0 \\
0 & I
\end{array}\right), \quad z \in \Omega,
$$

where $\Omega \subset \mathbb{C}$, and $E, F$ are holomorphic operator functions whose values are bijective mappings between suitable Banach spaces. This equivalence relation yields, in particular, the above-described spectral properties of $A$ and a simple analytic proof for (11). Furthermore, it clarifies the finite dimensional ingredient in the infinite dimensional dynamical system. Using the equivalence it is straightforward to construct the eigenfunctions and generalized eigenfunctions for the unbounded operator from the Jordan chains for the holomorphic matrix function $\Delta$. For this reason we call $\Delta$ a characteristic matrix for $A$ whenever (12) holds.

We also develop a general scheme to construct for a given unbounded op: erator $A$ a holomorphic matrix function $\Delta$ with the property that $A$ and $\Delta$ are related through an equivalence relation of the type (12). The class of unbounded operators to which this scheme may be applied includes many generators for evolutionary systems such as age-dependent population dynamics, a larger class of retarded functional differential equations than considered above, neutral functional differential equations, and the hyperbolic system of partial differential equations mentioned before. The abstract scheme yields a natural choice for the characteristic matrix associated with each of these problems. In this way, the multiplicity theorems in Levinger [22], Kappel and Wimmer [19], and Neves and Lin [26] appear as corollaries from the general construction, and new multiplicity theorems are derived for larger classes of functional differential equations and for the Lotka-McKendrick-von Förster equation (see Webb [31]) which provides a model for age-structured population dynamics.

\section{CHARACTERISTIC MATRICES FOR UNBOUNDED OPERATORS}

\section{I.1. Preliminaries}

Throughout this paper $X$ will denote a complex Banach space. The Banach algebra of bounded linear operators on $X$ endowed with the operator norm is denoted by $\mathscr{L}(X)$. Further, if $X$ and $Y$ denote complex Banach spaces, then $\mathscr{L}(X, Y)$ denotes the vector space of all bounded linear operators between $X$ and $Y$ endowed with the operator norm. 
I.1.1 Closed operators. In this section we summarize some elementary properties for unbounded closed operators and their spectral properties. (See, e.g., Taylor and Lay [29] and Gohberg, Goldberg and Kaashoek [10].) An unbounded linear operator $A$ on $X$ with domain $\mathscr{D}(A)$ will be denoted by $A(X \rightarrow X)$. An operator $A(X \rightarrow X)$ is called closed if and only if its graph $\{(x, A x): x \in \mathscr{D}(A)\}$ is closed in $X \times X$. Or equivalently, if $\mathscr{D}(A)$ endowed with the graph norm

$$
\|x\|_{A}:=\|x\|+\|A x\|
$$

becomes a Banach space $X_{A}$. Let $\tilde{A}: X_{A} \rightarrow X$ denote the bounded mapping induced by $A$ and $\widetilde{I}: X_{A} \rightarrow X$ the bounded embedding from $X_{A}$ into $X$.

Suppose $A(X \rightarrow X)$ is a closed operator, the resolvent set $\rho(A)$ of $A$ is the set of complex numbers $\lambda$ for which the resolvent $R(\lambda, A)=(\lambda I-A)^{-1}$ exists. The spectrum $\sigma(A)$ of $A$ is defined to be the complement of $\rho(A)$ in $\mathbb{C}$ and the point spectrum $\sigma_{p}(A) \subset \sigma(A)$ is the set of all $\lambda \in \mathbb{C}$ such that $\lambda I-A$ is not injective. Further, the resolvent set is open and

$$
z \mapsto R(z, A)
$$

is holomorphic from $\rho(A)$ into $\mathscr{L}(X)$. So, we can use contour integration to study isolated points in the spectrum of $A$.

If $\lambda$ is an isolated point of $\sigma(A)$, then the resolvent can be expanded in a Laurent series around $\lambda$

$$
R(z, A)=\sum_{l=-\infty}^{\infty}(z-\lambda)^{l} R_{l}
$$

for $0<|z-\lambda|<\delta$ and $\delta$ small. The coefficients $R_{l}$ are bounded linear operators on $X$ given by

$$
R_{l}=\frac{1}{2 \pi i} \int_{\Gamma_{\lambda}}(z-\lambda)^{-(l+1)} R(z, A) d z,
$$

where $\Gamma_{\lambda}=\{z:|z-\lambda|=\eta<\delta\}$. From the resolvent equation

$$
R(z, A)-R(\mu, A)=(\mu-z) R(z, A) R(\mu, A)
$$

it follows that the residue of $R(\cdot, A)$ at $\lambda$ is a projection

$$
P_{\lambda}=R_{-1}=\frac{1}{2 \pi i} \int_{\Gamma_{\lambda}} R(z, A) d z
$$

that is, $P_{\lambda}$ satisfies the equation $P_{\lambda}^{2}=P_{\lambda}$ and induces a direct sum decomposition

$$
X=\mathscr{M}_{\lambda} \oplus Q_{\lambda},
$$

where $\mathscr{M}_{\lambda}=\operatorname{Im} P_{\lambda}$ and $Q_{\lambda}=\operatorname{Ker} P_{\lambda}$ are closed $A$-invariant subspaces.

The direct sum (1.6) is called the spectral decomposition corresponding to $\lambda$, the subspace $\mathscr{M}_{\lambda}$ is called the generalized eigenspace at $\lambda$ and the projection $P_{\lambda}$ is called the Riesz spectral projection. The space $\mathscr{M}_{\lambda}$ is contained in $\mathscr{D}(A)$ and $A \mathscr{M}_{\lambda} \subset \mathscr{M}_{\lambda}$. Its dimension is called the algebraic multiplicity of $A$ at $\lambda$ and will be denoted by $M(A ; \lambda)$. If, in addition, $M(A ; \lambda)$ is finite, then $\lambda$ is called an eigenvalue of finite type. 
Let $\lambda$ be an eigenvalue of finite type of $A$. Then the generalized eigenspace $\mathscr{M}_{\lambda}$ has a basis

$$
y_{1,0}, \ldots, y_{1, \nu_{1}-1}, y_{2,0}, \ldots, y_{2, \nu_{2}-1}, y_{p, 0}, \ldots, y_{p, \nu_{p}-1},
$$

such that the matrix $A \mid \mathscr{M}_{\lambda}$ has Jordan normal form with $\lambda$ on the main diagonal. We shall call such a basis a canonical basis of eigenvectors and generalized eigenvectors for $A$ at $\lambda$. The partitioning of the basis (1.7) corresponds to the partitioning of the Jordan matrix in single Jordan blocks. The numbers $\nu_{1} \leq \nu_{2} \leq \cdots \leq \nu_{p}$ (which do not depend on the particular choice of the basis) are the sizes of the Jordan blocks, and they are called the partial multiplicities of the eigenvalue $\lambda$. The number of partial multiplicities is the geometric multiplicity of $\lambda$, i.e., $p=\operatorname{dim} \operatorname{Ker}(\lambda I-A)$. The largest partial multiplicity $\nu_{p}$ is equal to the smallest number $k$ such that

$$
\mathscr{M}_{\lambda}=\operatorname{Ker}\left((\lambda I-A)^{k}\right)
$$

and is called the ascent of $\lambda$. (See Taylor and Lay [29] for the general definition of this notion.)

Let $Z$ be a Banach space and $\widehat{A}(Z \rightarrow Z)$ an operator and assume $X \subset Z$. An operator $A(X \rightarrow X)$ is called the part of $\hat{A}$ if

$$
\mathscr{D}(A)=\{x \in X \cap \mathscr{D}(\widehat{A}): \widehat{A} x \in X\}, \quad A x=\widehat{A} x .
$$

The following proposition relates the spectra of $\hat{A}$ and the part of $\hat{A}$ in case $\mathscr{D}(\widehat{A}) \subset X$.

Proposition 1.1. Let $\widehat{A}(Z \rightarrow Z)$ and $\mathscr{D}(\widehat{A}) \subset X \subset Z$. If $A(X \rightarrow X)$ denotes the part of $\hat{A}$ in $X$, then

(i) $\sigma_{p}(\widehat{A})=\sigma_{p}(A)$ and for $\lambda \in \sigma_{p}(A)$

$$
\operatorname{Ker}\left((\lambda I-\widehat{A})^{k}\right)=\operatorname{Ker}\left((\lambda I-A)^{k}\right), \quad k=0,1,2, \ldots
$$

(ii) Furthermore, if $\lambda$ is an isolated point of $\sigma(A)$ and $\sigma(\widehat{A})$, then

$$
\left.P_{\lambda}(\widehat{A})\right|_{X}=P_{\lambda}(A), \quad \operatorname{Im} P_{\lambda}(\widehat{A})=\operatorname{Im} P_{\lambda}(A),
$$

that is, the algebraic multiplicity of $\lambda$ considered as an eigenvalue of $\hat{A}$ is the same as the algebraic multiplicity of $\lambda$ considered as an eigenvalue of $A$.

Proof. It is clear that $\sigma_{p}(A) \subset \sigma_{p}(\widehat{A})$. So, consider the case when $\lambda \in \sigma_{p}(\widehat{A})$, then there is a $\varphi \in \mathscr{D}(\widehat{A})$ such that $\hat{A} \varphi=\lambda \varphi$. Since $A$ is the part of $\hat{A}$, this shows that $\varphi$ must be in the domain of $A$ and hence $A \varphi=\lambda \varphi$. Thus $\lambda \in \sigma_{p}(A)$ and (i) follows using induction with respect to $k$. To prove (ii) note that if $\lambda$ is an isolated point of $\sigma(A)$ and $\sigma(\widehat{A})$, then $\left.P_{\lambda}(\widehat{A})\right|_{X}=P_{\lambda}(A)$ by definition of the part of $\widehat{A}$. So, (ii) follows from (i).

I.1.2 Equivalence and Jordan chains. Suppose $L: \Omega \rightarrow \mathscr{L}(X, Y)$ and $M: \Omega$ $\rightarrow \mathscr{L}\left(X^{\prime}, Y^{\prime}\right)$ are operator functions, holomorphic on an open set $\Omega$ in the complex plane $\mathbb{C}$. The operator functions $L$ and $M$ are called equivalent on 
$\Omega$ (see [9]) if there exist operator functions $E: \Omega \rightarrow \mathscr{L}\left(X^{\prime}, X\right)$ and $F: \Omega \rightarrow$ $\mathscr{L}\left(Y, Y^{\prime}\right)$, whose values are bijective operators, such that,

$$
M(z)=F(z) L(z) E(z), \quad z \in \Omega .
$$

Let $L: \Omega \rightarrow \mathscr{L}(X, Y)$ be a holomorphic operator function. A point $\lambda_{0}$ is called a characteristic value of $L$ if there exists a vector $x_{0} \in X, x_{0} \neq 0$, such that, $L\left(\lambda_{0}\right) x_{0}=0$. An ordered set $\left(x_{0}, x_{1}, \ldots, x_{k-1}\right)$ of vectors in $X$ is called a Jordan chain for $L$ at $\lambda_{0}$ if $x_{0} \neq 0$ and

$$
L(z)\left[x_{0}+\left(z-\lambda_{0}\right) x_{1}+\cdots+\left(z-\lambda_{0}\right)^{k-1} x_{k-1}\right]=O\left(\left(z-\lambda_{0}\right)^{k}\right) .
$$

The number $k$ is called the length of the chain and the maximal length of the chain starting with $x_{0}$ is called the rank of $x_{0}$. The holomorphic function

$$
\sum_{l=0}^{k-1}\left(z-\lambda_{0}\right)^{l} x_{l}
$$

in (1.9) is called a root function of $L$ corresponding to $\lambda_{0}$.

Proposition 1.2. If two holomorphic operator functions $L$ and $M$ are equivalent, then there is a one-to-one correspondence between their Jordan chains.

Proof. The equivalence relation (1.1) is symmetric, and thus it suffices to show that Jordan chains for $L$ yield Jordan chains for $M$. If $\left(x_{0}, \ldots, x_{k-1}\right)$ is a Jordan chain for $L$ at $\lambda_{0}$, then

$$
\begin{aligned}
& E(z)^{-1}\left(x_{0}+\left(z-\lambda_{0}\right) x_{1}+\cdots+\left(z-\lambda_{0}\right)^{k-1} x_{k-1}\right) \\
& \quad=y_{0}+\left(z-\lambda_{0}\right) y_{1}+\cdots+\left(z-\lambda_{0}\right)^{k-1} y_{k-1}+\text { h.o.t. }
\end{aligned}
$$

and $\left(y_{0}, \ldots, y_{k-1}\right)$ is a Jordan chain for $M$ at $\lambda_{0}$. Furthermore, the equivalence yields that the null spaces $\operatorname{Ker}\left(L\left(\lambda_{0}\right)\right)$ and $\operatorname{Ker}\left(M\left(\lambda_{0}\right)\right)$ are isomorphic and this shows the proposition.

Throughout this paper $\Delta: \Omega \rightarrow \mathscr{L}\left(\mathbb{C}^{n}\right)$ will denote a holomorphic matrix function. If the determinant of $\Delta$ is not identically zero, then we define $m(\lambda, \Delta)$ to be the order of $\lambda$ as zero of $\operatorname{det} \Delta$ and $k(\lambda, \Delta)$ to be the order of $\lambda$ as pole of operator function $\Delta^{-1}$.

Let $\lambda_{0}$ be an isolated characteristic value of $\Delta$, then the Jordan chains for $\Delta$ at $\lambda_{0}$ have finite rank and we can organize the chains according to the procedure described by Gohberg and Sigal [10]. Choose an eigenvector, say $x_{1,0}$, with maximal rank, say $r_{1}$. Next, choose a Jordan chain $\left(x_{1,0}, \ldots, x_{1, r_{1}-1}\right)$ of length $r_{1}$ and let $N_{1}$ be the complement in $\operatorname{Ker}\left(\Delta\left(\lambda_{0}\right)\right)$ of the subspace spanned by $x_{1,0}$. In $N_{1}$ we choose an eigenvector $x_{2,0}$ of maximal rank, say $r_{2}$, and let $\left(x_{2,0}, \ldots, x_{2, r_{2}-1}\right)$ be a corresponding Jordan chain of length $r_{2}$. We continue as follows, let $N_{2}$ be the complement in $N_{1}$ of the subspace spanned by $x_{2,0}$ and replace $N_{1}$ by $N_{2}$ in the above-described procedure.

In this way, we obtain a basis $\left\{x_{1,0}, \ldots, x_{p, 0}\right\}$ of $\operatorname{Ker}\left(\Delta\left(\lambda_{0}\right)\right)$ and a corresponding canonical system of Jordan chains

$$
x_{1,0}, \ldots, x_{1, r_{1}-1}, x_{2,0}, \ldots, x_{2, r_{2}-1}, x_{p, 0}, \ldots, x_{p, r_{p}-1}
$$

for $\Delta$ at $\lambda_{0}$.

It is easy to see that the rank of any eigenvector $x_{0}$ corresponding to the characteristic value $\lambda_{0}$ is always equal to one of the $r_{j}$ for $1 \leq j \leq p$. Thus, 
the integers $r_{1}, \ldots, r_{p}$ do not depend on the particular choices made in the procedure described above and are called the zero-multiplicities of $\Delta$ at $\lambda_{0}$. Their sum $r_{1}+\cdots+r_{p}$ is called the algebraic multiplicity of $\Delta$ at $\lambda_{0}$ and will be denoted by $M\left(\Delta\left(\lambda_{0}\right)\right)$.

In the linear case $\Delta(z)=z I-A$, a Jordan chain $\left(x_{0}, \ldots, x_{k-1}\right)$ for $\Delta$ at $\lambda_{0}$ satisfies

$$
\begin{gathered}
\left(A-\lambda_{0}\right) x_{0}=0 \\
\left(A-\lambda_{0}\right) x_{1}=x_{0} \\
\vdots \\
\left(A-\lambda_{0}\right) x_{k-1}=x_{k-2}
\end{gathered}
$$

and hence $\left\{\left(x_{i, 0}, \ldots, x_{i, r_{i}-1}\right) \mid i=1,2, \ldots, p\right\}$ is a canonical basis of eigenvectors and generalized eigenvectors for $A$ at $\lambda_{0}$. Thus, $M\left(\Delta\left(\lambda_{0}\right)\right)$ equals $M(A ; \lambda)$, the algebraic multiplicity of $A$ at $\lambda_{0}$, and the definition of the algebraic multiplicity using Jordan chains is a proper extension to the nonlinear case. In general, however, the system of Jordan chains for $\Delta$ at $\lambda_{0}$ is not a basis for the generalized null space of $\Delta\left(\lambda_{0}\right)$ and hence,

$$
\operatorname{dim} \operatorname{Ker}\left(\Delta\left(\lambda_{0}\right)^{k}\right) \neq M\left(\Delta\left(\lambda_{0}\right)\right), \quad k=\max _{1 \leq j \leq p} r_{j} .
$$

Next we recall the connection between the Jordan chains and the local Smith form for a holomorphic matrix function $\Delta: \Omega \rightarrow \mathscr{L}\left(\mathbb{C}^{n}\right)$ with $\operatorname{det} \Delta \not \equiv 0$. Let $\lambda_{0} \in \Omega$. Then there exist a neighbourhood $\mathscr{U}$ of $\lambda_{0}$, and holomorphic matrix functions $E$ and $F$ on $\mathscr{U}$ whose values are bijective operators such that

$$
\Delta(z)=F(z) D(z) E(z), \quad z \in \mathscr{U},
$$

where

$$
D(z)=\operatorname{diag}\left[\left(z-\lambda_{0}\right)^{\nu_{1}}, \ldots,\left(z-\lambda_{0}\right)^{\nu_{n}}\right], \quad z \in \mathscr{U} .
$$

The integers $\left\{\nu_{1}, \ldots, \nu_{n}\right\}$ are uniquely determined by $\Delta$ and the diagonal matrix $D$ is called the local Smith form for $\Delta$ at $\lambda_{0}$. This result is a special case of an abstract ring theoretical statement concerning matrices with entries in a principal ideal domain $R$ (see $\S \S 8$ and 10 in Chapter III of Jacobson [17]). To get the representation (1.11) one takes for $R$ the ring of all germs of complex functions holomorphic at $\lambda_{0}$.

For the local Smith form $D$ the Jordan chains are easily determined and it is clear that the set of zero multiplicities is given by $\left\{\nu_{1}, \ldots, \nu_{n}\right\}$. Hence, the equivalence (1.11) and Proposition 1.2 show that the algebraic multiplicity of $\Delta$ at $\lambda$ is given by

$$
M(\Delta(\lambda))=\sum_{l=1}^{n} \nu_{l} .
$$

On the other hand the equivalence yields

$$
\operatorname{det} \Delta(z)=\operatorname{det} F(z)\left(z-\lambda_{0}\right)^{\sum_{l=1}^{n} \nu_{l}} \operatorname{det} E(z)
$$

with $\operatorname{det} E\left(\lambda_{0}\right) \neq 0$ and $\operatorname{det} F\left(\lambda_{0}\right) \neq 0$. So, the multiplicity of $\lambda$ as zero of $\operatorname{det} \Delta$ equals the algebraic multiplicity of $\Delta$ at $\lambda$. 
An interesting application of the local Smith form to which we return in $\S$ I.2 is the following matrix valued multiplicity theorem:

$$
m\left(\lambda_{0}, \Delta\right)=\operatorname{tr}\left(\frac{1}{2 \pi i} \int_{\Gamma_{\lambda_{0}}} \Delta(z)^{-1} \frac{d}{d z} \Delta(z) d z\right),
$$

where $\Gamma_{\lambda_{0}}$ is a small circle surrounding only $\lambda_{0}$ of the discrete set $\sigma(A) \cap \Omega$ and $\operatorname{tr}(\cdot)$ denotes the trace operator. For (1.13) and more general versions of this identity see Gohberg and Sigal [11].

\section{I.2. Characteristic MATRICES AND SPECTRAL PROPERTIES}

Let $A$ be an unbounded operator on a Banach space $X$ and $\Omega$ an open set in the complex plane. We call a holomorphic matrix function $\Delta: \Omega \rightarrow \mathscr{L}\left(\mathbb{C}^{n}\right)$ a characteristic matrix for $A$ on $\Omega$ if there exist holomorphic operator functions $E: \Omega \rightarrow \mathscr{L}\left(\mathbb{C}^{n} \oplus X, Z \oplus X_{A}\right)$ and $F: \Omega \rightarrow \mathscr{L}\left(Z \oplus X, \mathbb{C}^{n} \oplus X\right)$, whose values are bijective operators, such that

$$
\left(\begin{array}{cc}
\Delta(z) & 0 \\
0 & I_{X}
\end{array}\right)=F(z)\left(\begin{array}{cc}
I_{Z} & 0 \\
0 & (z \tilde{I}-\tilde{A})
\end{array}\right) E(z), \quad z \in \Omega
$$

Here $\tilde{A}: X_{A} \rightarrow X$ and $\tilde{I}: X_{A} \rightarrow X$ are the bounded operators induced by $A$ and $I$. The operator function appearing in the left-hand side of (2.1) is called the $X$-extension of $\Delta$.

The next theorem justifies the terminology introduced above.

Theorem 2.1. Let $A$ be a closed unbounded operator on a Banach space $X$ and let $\Delta$ be a characteristic matrix for $A$ on $\Omega$ such that $\operatorname{det} \Delta \not \equiv 0$. Then

(i) the set $\sigma(A) \cap \Omega$ consists of eigenvalues of finite type and

$$
\sigma(A) \cap \Omega=\{z \in \Omega: \operatorname{det} \Delta(z)=0\} ;
$$

(ii) for $\lambda_{0} \in \sigma(A) \cap \Omega$, the partial multiplicities of $\lambda_{0}$ as an eigenvalue of $A$ are equal to the zero-multiplicities of $\Delta$ at $\lambda_{0}$;

(iii) for $\lambda_{0} \in \sigma(A) \cap \Omega$, the algebraic multiplicity of $\lambda_{0}$ as an eigenvalue of $A$ equals $m=m\left(\lambda_{0}, \Delta\right)$, the order of $\lambda_{0}$ as a zero of $\operatorname{det} \Delta$;

(iv) for $\lambda_{0} \in \sigma(A) \cap \Omega$, the ascent $k$ of $\lambda_{0}$ equals $k\left(\lambda_{0}, \Delta\right)$, the order of $\lambda_{0}$ as a pole of $\Delta^{-1}$ and

$$
\operatorname{dim} \operatorname{Ker}\left(\left(\lambda_{0} I-A\right)^{k}\right)=m \text {. }
$$

Proof. By definition of a characteristic matrix (2.2) holds. Define $\Sigma=\{z \in \Omega$ : $\operatorname{det} \Delta(z) \neq 0\}$, then

$$
\left(\begin{array}{cc}
I_{Z} & 0 \\
0 & (z \widetilde{I}-\tilde{A})^{-1}
\end{array}\right)=E(z)\left(\begin{array}{cc}
\Delta(z)^{-1} & 0 \\
0 & I_{X}
\end{array}\right) F(z), \quad z \in \Sigma .
$$

Since the values of $E$ and $F$ are bijective operators, $z \mapsto(z \widetilde{I}-\tilde{A})^{-1} \tilde{I}$ is finite meromorphic on $\Omega$, that is, for every $\lambda_{0} \in \sigma(A) \cap \Omega$ the Laurent expansion in a neighbourhood of $\lambda_{0}$ has the form

$$
(z I-A)^{-1}=(z \tilde{I}-\tilde{A})^{-1} \tilde{I}=\sum_{l=-n}^{\infty}\left(z-\lambda_{0}\right)^{l} R_{l}
$$


with $R_{-1}, \ldots, R_{-n}$ operators of finite rank. In particular, $R_{-1}$ has finite rank and (i) follows.

To prove (ii) let

$$
x_{1,0}, \ldots, x_{1, \nu_{1}-1}, x_{2,0}, \ldots, x_{2, \nu_{2}-1}, x_{p, 0}, \ldots, x_{p, \nu_{p}-1}
$$

with $\nu_{1} \leq \cdots \leq \nu_{p}$ be a canonical system of Jordan chains for $\Delta$ at $\lambda_{0}$. For $i=1, \ldots, p$ consider the polynomial

$$
\varphi_{i}(z)=x_{i, 0}+\left(z-\lambda_{0}\right) x_{i, 1}+\cdots+\left(z-\lambda_{0}\right)^{\nu_{i}-1} x_{i, \nu_{i}-1} .
$$

We know that

$$
\Delta(z) \varphi_{i}(z)=O\left(\left(z-\lambda_{0}\right)^{\nu_{i}}\right)
$$

Put

$$
\begin{aligned}
\psi_{i}(z) & =\left(\begin{array}{ll}
0 & \tilde{I}
\end{array}\right) E(z)\left(\begin{array}{c}
\varphi_{i}(z) \\
0
\end{array}\right) \\
& =y_{i, 0}+\left(z-\lambda_{0}\right) y_{i, 1}+\cdots+\left(z-\lambda_{0}\right)^{\nu_{i}-1} y_{i, \nu_{i}-1}+O\left(\left(z-\lambda_{0}\right)^{\nu_{i}}\right),
\end{aligned}
$$

From (2.6) and the equivalence (2.1), it follows that

$$
(z \tilde{I}-\tilde{A}) \psi_{i}(z)=O\left(\left(z-\lambda_{0}\right)^{\nu_{i}}\right),
$$

and thus

$$
\left(A-\lambda_{0}\right) y_{i, 0}=0,\left(A-\lambda_{0}\right) y_{i, 1}=y_{i, 0}, \ldots,\left(A-\lambda_{0}\right) y_{i, \nu_{i}-1}=y_{i, \nu_{i}-2}
$$

We shall prove that

$$
y_{1,0}, \ldots, y_{1, \nu_{1}-1}, y_{2,0}, \ldots, y_{2, \nu_{2}-1}, y_{p, 0}, \ldots, y_{p, \nu_{p}-1}
$$

is a canonical basis of eigenvectors and generalized eigenvectors of $A$ at $\lambda_{0}$. Note that $N: \mathbb{C}^{n} \rightarrow X_{A}$ defined by

$$
c \mapsto\left(\begin{array}{ll}
0 & \tilde{I}
\end{array}\right) E\left(\lambda_{0}\right)\left(\begin{array}{l}
c \\
0
\end{array}\right)
$$

maps $\operatorname{Ker} \Delta\left(\lambda_{0}\right)$ is a one-one way onto $\operatorname{Ker}\left(\lambda_{0} \tilde{I}-\tilde{A}\right)$. It follows that the vectors $y_{1,0}, \ldots, y_{p, 0}$ are linearly independent. But then we can use (2.7) to show that the set of vectors $(2.8)$ is linearly independent.

To finish the proof we show that $M\left(A ; \lambda_{0}\right)$, the algebraic multiplicity of $A$ at $\lambda_{0}$, is equal to $M\left(\Delta\left(\lambda_{0}\right)\right)$. Note that $M\left(A ; \lambda_{0}\right)$ equals the trace of the spectral projection $P_{\lambda_{0}}$. Further $P_{\lambda_{0}}$ restricted to $X_{A}$ equals the $\left(z-\lambda_{0}\right)^{-1}$ term in the expansion (2.5) and since the range of $P_{\lambda_{0}}$ is contained in $\mathscr{D}(A)$ we find

$$
\operatorname{tr} P_{\lambda_{0}}=\left.\operatorname{tr} P_{\lambda_{0}}\right|_{X_{A}}
$$

Let

$$
D(z)=\operatorname{diag}\left[\left(z-\lambda_{0}\right)^{\nu_{1}}, \ldots,\left(z-\lambda_{0}\right)^{\nu_{n}}\right]
$$

denote the local Smith form for $\Delta$ in a neighbourhood $\mathscr{U} \subset \Omega$ of $\lambda_{0}$, then there exist holomorphic matrix functions $E_{1}$ and $F_{1}$ on $\mathscr{U}$ whose values are bijective operators, such that,

$$
\Delta(z)=F_{1}(z) D(z) E_{1}(z), \quad z \in \mathscr{U} .
$$


Substituting (2.10) into (2.1) we find operator functions $E_{2}$ and $F_{2}$, holomorphic on $\mathscr{U}$, whose values are bijective operators, such that,

$$
\left(\begin{array}{cc}
D(z) & 0 \\
0 & I_{X}
\end{array}\right)=F_{2}(z)\left(\begin{array}{cc}
I_{Z} & 0 \\
0 & (z \widetilde{I}-\tilde{A})
\end{array}\right) E_{2}(z), \quad z \in \mathscr{U} .
$$

Let

$$
S[L]\left(\lambda_{0}\right)=\sum_{l=-n}^{-1}\left(z-\lambda_{0}\right)^{l} L_{l}
$$

denote the singular part in the Laurent expansion of a meromorphic operator function $L$ at $\lambda_{0}$. From (2.11) we derive

$$
\begin{aligned}
\operatorname{tr} S\left[D(z)^{-1}\right. & \left.\frac{d}{d z} D(z)\right]\left(\lambda_{0}\right) \\
= & \operatorname{tr} S\left[E_{2}(z)^{-1} L(z)^{-1} F_{2}(z)^{-1} \frac{d F_{2}}{d z}(z) L(z) E_{2}(z)\right]\left(\lambda_{0}\right) \\
& +\operatorname{tr} S\left[E_{2}(z)^{-1} L(z)^{-1} \frac{d L}{d z}(z) E_{2}(z)\right]\left(\lambda_{0}\right) \\
& +\operatorname{tr} S\left[E_{2}(z)^{-1} \frac{d E_{2}}{d z}(z)\right]\left(\lambda_{0}\right)
\end{aligned}
$$

where

$$
L(z)=\left(\begin{array}{cc}
I_{Z} & 0 \\
0 & (z \widetilde{I}-\widetilde{A})
\end{array}\right) .
$$

The operator function $z \mapsto E_{2}(z)^{-1} d / d z E_{2}(z)$ is holomorphic in a neighbourhood of $\lambda_{0}$. Therefore, the third term at the right-hand side does vanish. To analyze the remaining terms we need the following lemma (see Gohberg and Sigal [11]).

Lemma 2.2. Let $L$ and $M$ be finite meromorphic operator functions on $\Omega$. If $\lambda_{0} \in \Omega$ is a pole of $L$, then

$$
\operatorname{tr} S[L M]\left(\lambda_{0}\right)=\operatorname{tr} S[M L]\left(\lambda_{0}\right) .
$$

Because of the lemma the first term on the right-hand side of (2.12) equals

$$
\operatorname{tr} S\left[F_{2} \frac{d}{d z} F_{2}^{-1}\right]\left(\lambda_{0}\right)
$$

and hence does vanish. So,

$$
\operatorname{tr} S\left[D(z)^{-1} \frac{d}{d z} D(z)\right]\left(\lambda_{0}\right)=\operatorname{tr}\left[(z \widetilde{I}-\widetilde{A})^{-1} \widetilde{I}\right]\left(\lambda_{0}\right) .
$$

Now use that $D$ is a diagonal matrix given by (2.9) to calculate the right-hand side of (2.14). This yields

$$
\operatorname{tr} S\left[D(z)^{-1} \frac{d}{d z} D(z)\right]\left(\lambda_{0}\right)=\frac{\sum_{l=1}^{n} \nu_{l}}{\left(z-\lambda_{0}\right)} .
$$

By (2.5) this shows the formula

$$
\operatorname{tr} P_{\lambda_{0}}=M\left(\Delta\left(\lambda_{0}\right)\right),
$$


and completes the proof of (ii). To prove (iii), it remains to remark that, for a holomorphic matrix function $\Delta$ with $\operatorname{det} \Delta \neq 0$, the algebraic multiplicity $M\left(\Delta\left(\lambda_{0}\right)\right.$ equals the multiplicity of $\lambda_{0}$ as a zero of $\operatorname{det} \Delta$ (see $\left.\S I .2\right)$.

For the proof of (iv) note that the ascent of $\lambda_{0}$ equals the order of $\lambda_{0}$ as a pole of the resolvent $z \mapsto(z I-A)^{-1}$. But the order of a pole is invariant under equivalence and (iv) follows from (2.4).

\section{I.3. GENERAL SCHEME FOR CONSTRUCTING CHARACTERISTIC MATRICES}

In this section we develop a general scheme to construct characteristic matrices for a rather general class of unbounded operators. For this purpose we need auxiliary operators $D, L$ and $M$.

The operator $D(X \rightarrow X)$ is a closed linear operator acting in a complex Banach space $X$ and $D$ is assumed to satisfy the following two conditions:

(H1) $\mathcal{N}:=\operatorname{Ker}(D)$ is finite dimensional and $\mathscr{N} \neq\{0\}$;

(H2) the operator $D$ has a restriction $D_{0}(X \rightarrow X)$ such that
(1) (i) $\mathscr{D}(D)=\mathscr{N} \oplus \mathscr{D}\left(D_{0}\right)$,
(2) (ii) $\Omega:=\rho\left(D_{0}\right) \neq \varnothing$.

Apart from $D$ we need two bounded linear operators

$$
L: X_{D} \rightarrow \mathbb{C}^{n}, \quad M: X \rightarrow \mathbb{C}^{n} .
$$

Here $n=\operatorname{dim} \mathscr{N}$ and $X_{D}$ is the domain of $D$ endowed with the graph norm. One may think about $D$ as a maximal operator and about $L$ and $M$ as generalized boundary value operators.

With $D, L$ and $M$ as above we associate two operators $A(X \rightarrow X)$ and $\widehat{A}(Z \rightarrow Z)$, where

$$
Z=\mathbb{C}^{n} \times X=\left\{\left(\begin{array}{l}
c \\
\varphi
\end{array}\right): c \in \mathbb{C}^{n}, \varphi \in X\right\} .
$$

The definitions are as follows

$$
\mathscr{D}(A)=\{\varphi \in \mathscr{D}(D): M D \varphi=L \varphi\}, \quad A \varphi=D \varphi,
$$

and

$$
\begin{gathered}
\mathscr{D}(\widehat{A})=\left\{\left(\begin{array}{c}
c \\
\varphi
\end{array}\right) \in Z: \varphi \in \mathscr{D}(D), c=M \varphi\right\}, \\
\widehat{A}\left(\begin{array}{c}
c \\
\varphi
\end{array}\right)=\left(\begin{array}{c}
L \varphi \\
D \varphi
\end{array}\right) .
\end{gathered}
$$

The operators $A$ and $\widehat{A}$ are well-defined closed linear operators and are closely related. In fact, $A$ is similar to the part of $\widehat{A}$ in the graph of $M$ (see Lemma 3.3 below for the precise formulation). We shall refer to $A$ and $\widehat{A}$, respectively, as the first and second operator associated with $D, L$ and $M$.

Next, we define the candidate for the characteristic matrix function. Let $j: \mathbb{C}^{n} \rightarrow \mathcal{N}$ be some isomorphism, and set

$$
\Delta(z)=-(z M-L) D_{0}\left(z-D_{0}\right)^{-1} j, \quad z \in \Omega .
$$

Here $D_{0}$ is the operator appearing in Hypothesis $(\mathrm{H} 2)$ on $D$ and $\Omega$ is as defined in $(\mathrm{H} 2)$. 
Theorem 3.1. Suppose that $\widehat{A}(Z \rightarrow Z)$ is the second operator associated with $D, L$ and $M$. Then the matrix function $\Delta$ defined in (3.5) is a characteristic matrix for $\hat{A}$ and the equivalence is given by

$$
F(z)(z-\widehat{A}) E(z)=\left(\begin{array}{cc}
\Delta(z) & 0 \\
0 & I_{X}
\end{array}\right), \quad z \in \Omega,
$$

where $E: \Omega \rightarrow \mathscr{L}\left(Z, Z_{\widehat{A}}\right)$ and $F: \Omega \rightarrow \mathscr{L}(Z)$ are holomorphic operator functions whose values are bijective mappings. Furthermore, these operators and their inverses have the following representations

$$
\begin{gathered}
E(z)\left(\begin{array}{c}
c \\
\varphi
\end{array}\right)=\left(\begin{array}{c}
-M D_{0}\left(z-D_{0}\right)^{-1} j c+M\left(z-D_{0}\right)^{-1} \varphi \\
-D_{0}\left(z-D_{0}\right)^{-1} j c+\left(z-D_{0}\right)^{-1} \varphi
\end{array}\right), \\
E(z)^{-1}\left(\begin{array}{c}
M \psi \\
\psi
\end{array}\right)=\left(\begin{array}{c}
j^{-1}\left(\psi-D_{0}^{-1} D \psi\right) \\
(z-D) \psi
\end{array}\right),
\end{gathered}
$$

and

$$
\begin{aligned}
F(z)\left(\begin{array}{c}
c \\
\varphi
\end{array}\right) & =\left(\begin{array}{c}
c-z M\left(z-D_{0}\right)^{-1} \varphi+L\left(z-D_{0}\right)^{-1} \varphi \\
\varphi
\end{array}\right), \\
F(z)^{-1}\left(\begin{array}{c}
M \varphi \\
\varphi
\end{array}\right) & =\left(\begin{array}{c}
c+z M\left(z-D_{0}\right)^{-1} \varphi-L\left(z-D_{0}\right)^{-1} \varphi \\
\varphi
\end{array}\right) .
\end{aligned}
$$

Here $D_{0}$ is the operator appearing in the second hypothesis $(\mathrm{H} 2)$ on $D$.

The definition of the second operator $\hat{A}$ still makes sense when $M$ is only a bounded operator from $X_{D}$ into $\mathbb{C}^{n}$. Also, the characteristic matrix $\Delta$ remains well defined in this case. Therefore, in the proof of Theorem 3.1 we shall only use that $M$ acts as a bounded operator from $X_{D}$ into $\mathbb{C}^{n}$. (See the examples in Chapter II where we need this extension.)

Theorem 3.2. Suppose that $A$ is the first operator associated with $D, L$ and $M$ and let $\Delta$ be the matrix function defined by (3.5). Then $\rho(A) \cap \Omega \neq \varnothing$ if and only if $\operatorname{det} \Delta(z)$ does not vanish identically on $\Omega$, and in that case $\Delta$ is a characteristic matrix for $A$ on $\Omega$.

In the proof of Theorem 3.2 we shall construct explicitly an equivalence between extensions of $z-A$ and $\Delta(z)$ when $\rho(A) \cap \Omega \neq \varnothing$. To do this requires some preparations, which will come later. First we shall prove Theorem 3.1.

Proof of Theorem 3.1. From (H1) and (H2) we find for $z \in \Omega:=\rho\left(D_{0}\right)$

$$
X_{D}=\operatorname{Ker}(z-\widetilde{D}) \oplus X_{D_{0}},
$$

where $\widetilde{D}$ is the induced bounded operator from $X_{D}$ into $X$. To prove this direct sum choose $\varphi \neq 0$ such that $\varphi \in \operatorname{Ker}(z-\widetilde{D}) \cap X_{D_{0}}$, but then $\varphi$ must be an eigenvector of $D_{0}$ as well and this contradicts $z \in \Omega$. So,

$$
\operatorname{Ker}(z-\widetilde{D}) \cap X_{D_{0}}=\{0\} .
$$

Since for $z \in \Omega$ the mapping $\left(z-D_{0}\right)$ is onto, we derive that for every $\varphi \in X_{D}$, there exists a $\varphi_{1} \in X_{D_{0}}$ such that $(z-D) \varphi=\left(z-D_{0}\right) \varphi_{1}$. Thus

$$
\varphi=\left(\varphi-\varphi_{1}\right)+\varphi_{1},
$$

where $\varphi-\varphi_{1} \in \operatorname{Ker}(z-\widetilde{D})$ and this proves (3.7). 
In particular, there exists a holomorphic projection operator function $Q: \Omega$ $\rightarrow \mathscr{L}\left(X_{D}\right)$ such that

$$
Q(z)^{2}=Q(z) ; \quad \operatorname{Ker}(Q(z))=X_{D_{0}} ; \quad \operatorname{Im}(Q(z))=\operatorname{Ker}(z-\widetilde{D}) .
$$

In fact, $Q$ is given by

$$
Q(z) \varphi=\varphi-\left(z-D_{0}\right)^{-1}(z-\widetilde{D}) \varphi, \quad \varphi \in X_{D} .
$$

Further, since $\operatorname{Ker}(Q(z))$ does not depend on $z$, we have

$$
Q\left(z_{1}\right)=Q\left(z_{1}\right) Q\left(z_{2}\right), \quad z_{1}, z_{2} \in \Omega \text {. }
$$

In the sequel, we only need the action of $Q$ on the range of $j$. Since $\operatorname{Im}(j)=$ $\operatorname{Ker}(D)$,

$$
Q(z) j=j-z\left(z-D_{0}\right)^{-1} j=-D_{0}\left(z-D_{0}\right)^{-1} j .
$$

Hence, the characteristic matrix (3.5) is given by

$$
\Delta(z)=(z M-L) Q(z) j
$$

and this shows that $\Delta$ is well defined for $L, M: X_{D} \rightarrow \mathbb{R}^{n}$. Put $H: \Omega \rightarrow$ $\mathscr{L}\left(Z, X_{D}\right)$

$$
H(z)\left(\begin{array}{c}
c \\
\varphi
\end{array}\right)=Q(z) j c+\left(z-D_{0}\right)^{-1} \varphi .
$$

First we show that $H(z)$ is invertible and $H(z)^{-1}$ is given by

$$
H(z)^{-1} \psi=\left(\begin{array}{c}
j^{-1} Q(0) \psi \\
(z-D) \psi
\end{array}\right) .
$$

Define $K: \Omega \rightarrow \mathscr{L}\left(X_{D}, Z\right)$

$$
K(z) \psi=\left(\begin{array}{c}
j^{-1} Q(0) \psi \\
(z-D) \psi
\end{array}\right),
$$

then

Further

$$
K(z) H(z)\left(\begin{array}{c}
c \\
\varphi
\end{array}\right)=K(z)\left[Q(z) j c+\left(z-D_{0}\right)^{-1} \varphi\right] .
$$

and from (3.9)-(3.10)

$$
(z-D)\left[Q(z) j c+\left(z-D_{0}\right)^{-1} \varphi\right]=\varphi
$$

$$
\begin{aligned}
j^{-1} Q(0)\left[Q(z) j c+\left(z-D_{0}\right)^{-1} \varphi\right] & =j^{-1} Q(0) Q(z) j c+j^{-1}\left(z-D_{0}\right)^{-1} \varphi \\
& =j^{-1} Q(0) j c=c .
\end{aligned}
$$

Hence

and since $Q(z)=Q(z) Q(0)$

$$
K(z) H(z)\left(\begin{array}{c}
c \\
\varphi
\end{array}\right)=\left(\begin{array}{c}
c \\
\varphi
\end{array}\right)
$$

$$
\begin{aligned}
H(z) K(z) \psi & =H(z)\left(\begin{array}{c}
j^{-1} Q(0) \psi \\
(z-D) \psi
\end{array}\right) \\
& =Q(z) j^{-1} Q(0) \psi+\left(z-D_{0}\right)^{-1}(z-D) \psi \\
& =Q(z) j^{-1} Q(0)[I-Q(z)+Q(z)] Q(0) \psi+\psi-Q(z) \psi \\
& =Q(z) j^{-1} Q(0) Q(z) Q(0) \psi+\psi-Q(z) \psi \\
& =Q(z) Q(0) \psi+\psi-Q(z) \psi=\psi
\end{aligned}
$$


Consequently, the values of $H$ are invertible and $H(z)^{-1}=K(z)$ for $z \in \Omega$. Next, we define the operator function $E: \Omega \rightarrow \mathscr{L}\left(Z, Z_{\widehat{A}}\right)$ by

$$
E(z)\left(\begin{array}{l}
c \\
\varphi
\end{array}\right)=J H(z)\left(\begin{array}{l}
c \\
\varphi
\end{array}\right)
$$

where $J: X_{D} \rightarrow Z$ is given by

$$
J \varphi=\left(\begin{array}{c}
M \varphi \\
\varphi
\end{array}\right)
$$

Then $E$ is a holomorphic operator function whose values are invertible and

$$
E(z)^{-1}\left(\begin{array}{c}
M \psi \\
\psi
\end{array}\right)=H(z)^{-1} \psi=\left(\begin{array}{c}
j^{-1} Q(0) \psi \\
(z-D) \psi
\end{array}\right) .
$$

The action of $(z-\widehat{A})$ on $E(z)\left(\begin{array}{l}c \\ \varphi\end{array}\right)$ is given by

$$
\begin{aligned}
(z-\widehat{A}) E(z)\left(\begin{array}{c}
c \\
\varphi
\end{array}\right) & =(z-\widehat{A})\left(\begin{array}{c}
-M D_{0}\left(z-D_{0}\right)^{-1} j c+M\left(z-D_{0}\right)^{-1} \varphi \\
D_{0}\left(z-D_{0}\right)^{-1} j c+\left(z-D_{0}\right)^{-1} \varphi
\end{array}\right) \\
& =\left(\begin{array}{c}
\Delta(z) c+z M\left(z-D_{0}\right)^{-1} \varphi-L\left(z-D_{0}\right)^{-1} \varphi \\
\varphi
\end{array}\right),
\end{aligned}
$$

where $\Delta: \Omega \rightarrow \mathscr{L}\left(\mathbb{C}^{n}\right)$ denotes the candidate characteristic matrix given by (3.5). Finally, we define the operator function $F: \Omega \rightarrow \mathscr{L}(Z)$ by

$$
F(z)\left(\begin{array}{c}
c \\
\varphi
\end{array}\right)=\left(\begin{array}{c}
c-z M\left(z-D_{0}\right)^{-1} \varphi+L\left(z-D_{0}\right)^{-1} \varphi \\
\varphi
\end{array}\right)
$$

and this completes the equivalence

$$
F(z)(z-\widehat{A}) E(z)=\left(\begin{array}{cc}
\Delta(z) & 0 \\
0 & I
\end{array}\right)
$$

To prove Theorem 3.2, we need to describe the relation between $A$ and $\widehat{A}$. Let $Y$ be the closed subspace of $Z$ defined by the graph of $M$, i.e.,

$$
Y=\left\{\left(\begin{array}{c}
c \\
\varphi
\end{array}\right): c=M \varphi\right\}
$$

Note that $\mathscr{D}(\widehat{A}) \subset Y$.

Lemma 3.3. Suppose that $A$ and $\widehat{A}$ are the first and second operator, respectively, associated with $D, L$ and $M$. Then $A$ is similar to the part of $\widehat{A}$ in $Y$. More precisely, let $J: X \rightarrow Y$ be the isomorphism between $X$ and $Y$ given by

$$
J \varphi=\left(\begin{array}{c}
M \varphi \\
\varphi
\end{array}\right)
$$

then $J \mathscr{D}(A) \subset \mathscr{D}(\widehat{A})$ and $J A J^{-1}$ is precisely the part of $\widehat{A}$ in $Y$.

Proof. By definition, we have to verify the following property

and

$$
\mathscr{D}\left(J A J^{-1}\right)=\left\{\left(\begin{array}{c}
c \\
\varphi
\end{array}\right) \in \mathscr{D}(\widehat{A}): \widehat{A}\left(\begin{array}{c}
c \\
\varphi
\end{array}\right) \in Y\right\}
$$




$$
\widehat{A}\left(\begin{array}{c}
c \\
\varphi
\end{array}\right)=J A J^{-1}\left(\begin{array}{c}
c \\
\varphi
\end{array}\right) \quad \text { for }\left(\begin{array}{c}
c \\
\varphi
\end{array}\right) \in \mathscr{D}\left(J A J^{-1}\right),
$$

but this is immediate from the definitions.

Consequently, Proposition 1.1 yields that the point spectra of $A$ and $\widehat{A}$ are the same and have the same spectral data.

Next we analyze the property that $A$ is similar to the part of $\widehat{A}$ a little further when $\operatorname{det} \Delta \not \equiv 0$. Put

$$
Z=Y \oplus N: \quad\left(\begin{array}{c}
c \\
\varphi
\end{array}\right)=\left(\begin{array}{c}
M \varphi \\
\varphi
\end{array}\right)+\left(\begin{array}{c}
c-M \varphi \\
0
\end{array}\right)
$$

and define operators $T_{1}(Z \rightarrow Z)$ and $T_{2}(Z \rightarrow Z)$ by

$$
T_{1}\left(\begin{array}{c}
c \\
\varphi
\end{array}\right)=\left(\begin{array}{c}
M D \varphi \\
D \varphi
\end{array}\right) ; \quad T_{2}\left(\begin{array}{c}
c \\
\varphi
\end{array}\right)=\left(\begin{array}{c}
L \varphi-M D \varphi \\
0
\end{array}\right) .
$$

Then $\widehat{A}=T_{1}+T_{2}$ and

$$
\mathscr{D}(A)=\mathscr{D}(\widehat{A}) \cap \operatorname{Ker}\left(T_{2}\right) .
$$

Lemma 3.4. If $\operatorname{det} \Delta \not \equiv 0$ on $\Omega$, then $T_{2}$ maps $Z$ onto $N$.

Proof. From the proof of Theorem 3.1 it follows that the characteristic matrix is given by

$$
\Delta(z)=(z M-L) Q(z) j,
$$

but on the range of $Q(z)$ the mapping $z M$ acts as $M D$. Thus,

$$
\Delta(z)=(M D-L) Q(z) j
$$

and the condition $\operatorname{det} \Delta \not \equiv 0$ on $\Omega$ implies that there is a $z_{0} \in \Omega$ such that $\Delta\left(z_{0}\right)$ is invertible. From (3.10) we conclude that the mapping $Q(z) j$ is a oneto-one mapping. This shows that $(M D-L)$ is onto $\mathbb{C}^{n}$ and this proves the lemma.

If $T_{2}^{+}: N \rightarrow Z$ denotes the right inverse of $T_{2}$, then by the lemma $T_{2}^{+}$can be represented as

$$
\left(\begin{array}{l}
c \\
0
\end{array}\right) \rightarrow\left(\begin{array}{c}
-M Q\left(z_{0}\right) j \Delta\left(z_{0}\right)^{-1} c \\
-Q\left(z_{0}\right) j \Delta\left(z_{0}\right)^{-1} c
\end{array}\right)
$$

Define the projection $P=\left.T_{2}^{+} T_{2}\right|_{Y}: Y \rightarrow \tilde{N}$

$$
P\left(\begin{array}{c}
M \varphi \\
\varphi
\end{array}\right)=\left(\begin{array}{c}
M Q\left(z_{0}\right) j \Delta\left(z_{0}\right)^{-1}(M D-L) \varphi \\
Q\left(z_{0}\right) j \Delta\left(z_{0}\right)^{-1}(M D-L) \varphi
\end{array}\right),
$$

where $\tilde{N}$ denotes the range of $T_{2}^{+}$and $z_{0}$ is such that $\operatorname{det} \Delta\left(z_{0}\right) \neq 0$. Then $\operatorname{Im}(I-P)=Y_{J A J^{-1}}$ and $P$ is the projection associated with the direct sum

$$
Z_{\widehat{A}}=Y_{J A J^{-1}} \oplus \tilde{N}
$$

Consequently $\widehat{A}: Y_{J A J^{-1}} \oplus \widetilde{N} \rightarrow Y \oplus N$ can be represented by an operator matrix

$$
\left(\begin{array}{cc}
J A J^{-1} & T_{10} \\
0 & T_{20}
\end{array}\right)
$$


where

$$
T_{10}=\left.T_{1}\right|_{\widetilde{N}}: \tilde{N} \rightarrow Y, \quad T_{20}=\left.T_{2}\right|_{\widetilde{N}}: \tilde{N} \rightarrow N .
$$

With respect to the above direct sum the identity operator has the operator matrix representation

$$
I=\left(\begin{array}{cc}
I_{Y_{J A J-1}} & I_{\widetilde{N}} \\
0 & 0
\end{array}\right)
$$

and we have proved the following lemma.

Lemma 3.5. If $\operatorname{det} \Delta \not \equiv 0$ on $\Omega$, then $T_{20}: \tilde{N} \rightarrow N$ is bijective and

$$
z-\widehat{A}=\left(\begin{array}{cc}
I_{Y_{J A J^{-1}}} & z-T_{10} \\
0 & -T_{20}
\end{array}\right)\left(\begin{array}{cc}
z-J A J^{-1} & 0 \\
0 & I_{\widetilde{N}}
\end{array}\right) \text {. }
$$

Proof of Theorem 3.2. To construct the equivalence we use the projection $P$ defined by (3.15) and the equivalence for $z-\widehat{A}$ constructed in Theorem 3.1. Consider the $\mathbb{C}^{n}$-extension of $z-A$ and define the holomorphic operator function $\widetilde{E}: \Omega \rightarrow \mathscr{L}\left(Z, \mathbb{C}^{n} \times X_{A}\right)$ by

$$
\widetilde{E}(z)\left(\begin{array}{c}
c \\
\varphi
\end{array}\right)=\left(\begin{array}{c}
(M D-L)\left[Q(z) j c+\left(z-D_{0}\right)^{-1} \varphi\right] \\
\left(I-Q\left(z_{0}\right) j \Delta\left(z_{0}\right)^{-1}(M D-L)\right)\left[Q(z) j c+\left(z-D_{0}\right)^{-1} \varphi\right]
\end{array}\right) .
$$

Because of Lemma 3.5 the values of $\widetilde{E}$ are bijective mappings. Furthermore

$$
\begin{gathered}
\left(\begin{array}{cc}
I_{\mathbb{C}^{n}} & 0 \\
0 & z-A
\end{array}\right) \widetilde{E}(z)\left(\begin{array}{c}
c \\
\varphi
\end{array}\right) \\
=\left(\begin{array}{c}
(M D-L)\left[Q(z) j c+\left(z-D_{0}\right)^{-1} \varphi\right] \\
\varphi-(z-D) Q\left(z_{0}\right) j \Delta\left(z_{0}\right)^{-1}(M D-L)\left[Q(z) j c+\left(z-D_{0}\right)^{-1} \varphi\right]
\end{array}\right) .
\end{gathered}
$$

Finally, we define the holomorphic operator function $\widetilde{F}: \Omega \rightarrow \mathscr{L}(Z)$ by

$$
\widetilde{F}(z)\left(\begin{array}{c}
c \\
\varphi
\end{array}\right)=\left(\begin{array}{c}
c-(M D-L)\left(z-D_{0}\right)^{-1} \varphi \\
\varphi+\left(z-z_{0}\right) Q\left(z_{0}\right) j \Delta\left(z_{0}\right)^{-1} c
\end{array}\right) .
$$

Clearly, the values of $\widetilde{F}$ are bijective mappings and the equivalence is given by

$$
\widetilde{F}(z)\left(\begin{array}{cc}
I_{\mathbb{C}^{n}} & 0 \\
0 & z-A
\end{array}\right) \widetilde{E}(z)=\left(\begin{array}{cc}
\Delta(z) & 0 \\
0 & \widetilde{I}
\end{array}\right) .
$$

This completes the proof of Theorem 3.2.

Corollary 3.7. Let $\operatorname{det} \Delta \not \equiv 0$ on $\Omega$, and let $\lambda_{0}$ be a characteristic value of $\Delta$, and let

$$
\left\{\left(x_{i, 0}, \ldots, x_{i, k_{i}-1}\right) \mid i=1, \ldots, p\right\}
$$

be a canonical system of Jordan chains for $\Delta$ at $\lambda_{0}$. Then

$$
\begin{gathered}
\left\{y_{i, 0}, \ldots, y_{i, k_{i}-1} \mid i=1, \ldots, p\right\} \\
\left\{\left(\begin{array}{c}
M y_{i, 0} \\
y_{i, 0}
\end{array}\right), \ldots,\left(\begin{array}{c}
M y_{i, k_{i}-1} \\
y_{i, k_{i}-1}
\end{array}\right) \mid i=1, \ldots, p\right\},
\end{gathered}
$$

are canonical bases of eigenvectors and generalized eigenvectors for $A$ and $\widehat{A}$, respectively, at $\lambda_{0}$. Here

$$
y_{i, \nu}=\sum_{l=0}^{\nu} D_{0}\left(D_{0}-\lambda_{0}\right)^{-l-1} j\left(x_{i, \nu-l}\right)
$$


where $j$ denotes some isomorphism from $\mathbb{C}^{n}$ onto $\mathcal{N}, \nu=0, \ldots, k_{i}-1$ and $i=1, \ldots, p$.

Proof. Let $\left(x_{0}, \ldots, x_{k-1}\right)$ be a Jordan chain for $\Delta$ at $\lambda_{0}$ of length $k$. Because of the equivalence (3.6) it follows that

$$
J H(z)\left(\begin{array}{c}
x_{0}+x_{1}(z-\lambda)+\cdots+x_{k-1}(z-\lambda)^{k-1} \\
0
\end{array}\right)
$$

is a root function for $(z-\widehat{A})$ at $\lambda_{0}$. Here $J: X_{D} \rightarrow Z$ is given by (3.12) and $H: \Omega \rightarrow \mathscr{L}\left(Z, X_{D}\right)$ is given by (3.11). From the proof of part (ii) of Theorem 2.1 it follows that we have to expand (3.19) up to order $k$ in a neighbourhood of $\lambda$. But

$$
H(z)\left(\begin{array}{l}
c \\
0
\end{array}\right)=-D_{0}\left(z-D_{0}\right)^{-1} j c
$$

and

$$
\begin{aligned}
\left(z-D_{0}\right)^{-1} & =\left(\lambda_{0}-D_{0}\right)^{-1}\left[I-\left(z-\lambda_{0}\right)\left(D_{0}-\lambda_{0}\right)^{-1}\right]^{-1} \\
& =-\sum_{l=0}^{\infty}\left(z-\lambda_{0}\right)^{l}\left(D_{0}-\lambda_{0}\right)^{-l-1}
\end{aligned}
$$

so that the corollary follows from the results of $\S \mathbf{I} .2$.

\section{I.4. ORDINARY DIFFERENTIAL EQUATIONS}

As a first illustration of our results consider the following boundary value problem

$$
\begin{aligned}
& \frac{d \varphi}{d x}(x)+q(x) \varphi(x)=f(x) \text { for } a \leq x \leq b, \\
& L_{1} \varphi(a)+L_{2} \varphi(b)=\varphi_{0}
\end{aligned}
$$

Here $L_{1}, L_{2}$ are constant $n \times n$ matrices, $q$ is an $n \times n$ matrix valued function which is continuous on $[a, b]$, and $\varphi_{0}$ is a given vector in $\mathbb{C}^{n}$. The problem is to find a vector valued solution $\varphi \in W^{1, p}[a, b]$, the Sobolev space of all functions in $L^{p}[a, b]$ for which the distributional derivative is also in $L^{p}[a, b]$, such that $\varphi$ satisfies the equations in (4.1). More generally, consider the following first order differential operator $A\left(L^{p}[a, b] \rightarrow L^{p}[a, b]\right)$

$$
\begin{aligned}
\mathscr{D}(A) & =\left\{\varphi \in L^{p}[a, b]: \varphi \in W^{1, p}[a, b], L \varphi=0\right\}, \\
A \varphi & =\frac{d \varphi}{d x}+q(\cdot) \varphi,
\end{aligned}
$$

where $L: W^{1, p}[a, b] \rightarrow \mathbb{C}^{n}$ is a bounded linear mapping.

Lemma 4.1. Let $D\left(L^{p}[a, b] \rightarrow L^{p}[a, b]\right)$ be the operator defined by

$$
\begin{aligned}
\mathscr{D}(D) & =\left\{\varphi \in L^{p}[a, b]: \varphi \in W^{1, p}[a, b]\right\}, \\
(D \varphi)(x) & =\frac{d \varphi}{d x}(x)+q(x) \varphi(x) .
\end{aligned}
$$

Then $D$ satisfies the hypothesis $(\mathrm{H} 1),(\mathrm{H} 2)$ with $\Omega=\mathbb{C}$. Furthermore, the operator $A$ defined by (4.2) is the first operator associated with $D, L$ and $M$, where $M=0$. 
Proof. Put $X=L^{p}[a, b]$. To find the kernel of $D$ we solve the equation

$$
\frac{d \varphi}{d x}(x)+q(x) \varphi(x)=0, \quad a \leq x \leq b .
$$

The solution is

$$
\varphi(x)=Y(x, a) \varphi(a), \quad a \leq x \leq b,
$$

where $Y(\cdot, a)$ denotes the fundamental matrix solution of (4.3) with $Y(a, a)$ $=I_{n}$. It follows that $\mathscr{N}=\operatorname{Ker} D$ has dimension $n$. For $D_{0}$ we take the operator $D_{0}(X \rightarrow X)$ defined by

$$
\mathscr{D}\left(D_{0}\right)=\{\varphi \in \mathscr{D}(D): \varphi(a)=0\}, \quad D_{0} \varphi=D \varphi .
$$

Clearly, $\mathscr{D}(D)=\mathscr{N} \oplus \mathscr{D}\left(D_{0}\right)$. To find $\Omega=\rho\left(D_{0}\right)$ we first note that the equation

$$
\frac{d \varphi}{d x}(x)+q(x) \varphi(x)=z \varphi(x), \quad a \leq x \leq b,
$$

is solvable for each $z \in \mathbb{C}$ and its solution is given by

$$
\varphi(x)=e^{z(x-a)} Y(x, a) \varphi(a) .
$$

It follows that $\rho\left(D_{0}\right)=\mathbb{C}$ and the resolvent of $D_{0}$ is given by

$$
\left(\left(z-D_{0}\right)^{-1} \psi\right)(x)=-\int_{a}^{x} e^{z(x-s)} Y(x, s) \psi(s) d s, \quad a \leq x \leq b .
$$

Therefore $D$ satisfies the hypotheses $(\mathrm{H} 1),(\mathrm{H} 2)$ and $\Omega=\mathbb{C}$.

For the case considered here the Banach space $Z$ equals $\mathbb{C}^{n} \times L^{p}[a, b]$ and the second operator $\widehat{A}(Z \rightarrow Z)$ associated with the triple $D, L$ and $M$ is given by

$$
\mathscr{D}(\widehat{A})=\left\{\left(\begin{array}{c}
c \\
\varphi
\end{array}\right) \in Z: \varphi \in \mathscr{D}(D), c=0\right\}, \quad \hat{A}\left(\begin{array}{c}
c \\
\varphi
\end{array}\right)=\left(\begin{array}{c}
L \varphi \\
D \varphi
\end{array}\right) .
$$

Thus, we can apply Theorem 3.1 to obtain

Theorem 4.2. The matrix function $\Delta: \mathbb{C} \rightarrow \mathscr{L}\left(\mathbb{C}^{n}\right)$

$$
\Delta(z)=L\left(e^{z \cdot} Y(\cdot, a)\right),
$$

where $Y(\cdot, a)$ is a fundamental matrix solution of

$$
\frac{d \varphi}{d x}(x)+q(x) \varphi(x)=0
$$

is a characteristic matrix for $\hat{A}$ defined by (4.5) and the equivalence is given by

$$
\left(\begin{array}{cc}
\Delta(z) & 0 \\
0 & I
\end{array}\right)=F(z)(z-\widehat{A}) E(z),
$$

where $E: \mathbb{C} \rightarrow \mathscr{L}\left(Z, Z_{\widehat{A}}\right)$ is given by

$$
E(z)\left(\begin{array}{l}
c \\
\varphi
\end{array}\right)=\left(\begin{array}{l}
0 \\
\psi
\end{array}\right)
$$

with

$$
\psi(x)=e^{z x} Y(x, a) c-\int_{a}^{x} e^{z(x-s)} Y(x, s) \varphi(s) d s,
$$




$$
E(z)^{-1}\left(\begin{array}{c}
0 \\
\psi
\end{array}\right)=\left(\begin{array}{c}
\psi(0) \\
(z-D) \psi
\end{array}\right)
$$

and $F: \mathbb{C} \rightarrow \mathscr{L}(Z)$ is given by

$$
\begin{aligned}
F(z)\left(\begin{array}{l}
c \\
\varphi
\end{array}\right) & =\left(\begin{array}{c}
c+L\left(z-D_{0}\right)^{-1} \varphi \\
\varphi
\end{array}\right), \\
F(z)^{-1}\left(\begin{array}{l}
c \\
\varphi
\end{array}\right) & =\left(\begin{array}{c}
c-L\left(z-D_{0}\right)^{-1} \varphi \\
\varphi
\end{array}\right) .
\end{aligned}
$$

Proof. Let $D$ and $D_{0}$ be as in the proof of Lemma 4.1. Define $j: \mathbb{C}^{n} \rightarrow \mathscr{N}$, $\mathscr{N}=\operatorname{Ker} D$ by $c \mapsto Y(\cdot, a) c$, and put

$$
\Delta(z)=L\left(-j+z\left(z-D_{0}\right)^{-1} j\right) .
$$

From Theorem 3.1 we know that $\Delta$ is a characteristic matrix function for $\widehat{A}$. To verify the concrete representation (4.6) for $\Delta$, we use the resolvent formula (4.4)

$$
\begin{aligned}
\Delta(z) & =L\left(-j+z\left(z-D_{0}\right)^{-1} j\right) \\
& =L\left(-j-z \int_{a}^{x} e^{z(x-s)} Y(x, s) Y(s, a) d s\right) \\
& =L\left(-Y(x, a)-z \int_{a}^{x} e^{-z s} d s e^{z x} Y(x, a)\right) \\
& =L\left(e^{z} \cdot Y(\cdot, a)\right) .
\end{aligned}
$$

The explicit equivalence relation (4.7) follows from Theorem 3.1 by similar calculations.

If the boundary value operator $L$ is given by

$$
L \varphi=L_{1} \varphi(a)+L_{2} \varphi(b),
$$

where $L_{1}$ and $L_{2}$ are as in (4.1), then Theorem 4.2 reduces to Theorem 1.1 in Kaashoek [18] (see also Mennicken and Möller [24]).

If $\operatorname{det} \Delta \not \equiv 0$, then Theorem 3.2 shows that $\Delta$ actually is a characteristic matrix for $A$ itself and we have the following corollary.

Corollary 4.3. If $\operatorname{det} \Delta \not \equiv 0$, then the spectrum of $A\left(L^{p}[a, b] \rightarrow L^{p}[a, b]\right) d e$ fined by (4.2) consists of eigenvalues of finite type only,

$$
\sigma(A)=\{\lambda: \operatorname{det} \Delta(\lambda)=0\} .
$$

For $\lambda \in \sigma(A)$, the algebraic multiplicity of the eigenvalue $\lambda$ equals the order of $\lambda$ as a zero of $\operatorname{det} \Delta$, the partial multiplicities of the eigenvalue $\lambda$ are equal to the zero-multiplicities of $\lambda$ as a characteristic value of $\Delta$, and the largest partial multiplicity (ascent) of $\lambda$ equals the order of $\lambda$ as a pole of $\Delta^{-1}$. Furthermore, a canonical basis of eigenvectors and generalized eigenvectors for $A$ at $\lambda$ may be obtained in the following way: If $\left\{\left(\gamma_{i, 0}, \ldots, \gamma_{i, k_{i}-1}\right) \mid i=1, \ldots, p\right\}$ is a canonical system of Jordan chains for $\Delta$ at $\lambda \in \Omega$, then $\left\{\chi_{i, 0} \ldots, \chi_{i, k_{i}-1} \mid i=\right.$ $1, \ldots, p\}$, where

$$
\chi_{i, \nu}(x)=e^{\lambda x} \sum_{l=0}^{\nu} Y(x, a) \gamma_{i, \nu-l} \frac{x^{l}}{l !},
$$

yields a canonical basis for $A$ at $\lambda$. 
Remark 4.4. The construction carried out in this section may also be applied to problems where the spectral parameter $\lambda$ appears in a nonlinear way. For examples in this direction see Kaashoek [18], and Langer and Möller [21].

\section{APPLICATIONS TO EVOLUTIONARY SYSTEMS}

\section{II.1. FUNCTIONAL DIFFERENTIAL EQUATIONS}

The first class of examples concerns linear autonomous functional differential equations

$$
\dot{x}(t)=L x_{t}, \quad t \geq 0,
$$

where $L: X \rightarrow \mathbb{C}^{n}$ is a linear mapping from a Banach space $X$ into $\mathbb{C}^{n}$. Different choices for the mapping $L$ and the space $X$ lead to different type of functional differential equations.

II.1.1 Retarded functional differential equations. At first we consider the finite delay case, that is, the state space $X$ equals $C[-h, 0]$, the space of continuous functions on $[-h, 0]$ provided with the supremum norm. (See Hale [13].)

The "functional" $L$ can be represented in a unique way as a RiemannStieltjes integral

$$
L \varphi=\int_{0}^{h} d \zeta(\theta) \varphi(-\theta),
$$

where $\zeta$ is an $n \times n$-matrix of bounded variation on $[0, h]$ which is normalized such that $\zeta(0)=0, \zeta$ is left continuous on $[0, h]$ and constant for $t \geq h$. Further the state $x_{t}$ is given by

$$
x_{t}(\theta)=x(t+\theta) \text { for }-h \leq \theta \leq 0
$$

and (1.1) can be written in the form

$$
\dot{x}(t)=\int_{0}^{h} d \zeta(\theta) x(t-\theta), \quad t \geq 0 .
$$

The initial value problem with $x_{0}=\varphi$ and $\varphi \in C[-h, 0]$ is well posed, that is, for any given $\varphi \in C[-h, 0]$ a solution $x(\cdot ; \varphi)$ exists on $[0, \infty)$ and is unique. Thus, the solution operator $T(t): C[-h, 0] \rightarrow C[-h, 0]$

$$
T(t) \varphi=x_{t}(\cdot ; \varphi)
$$

is a semigroup of bounded linear operators and it is easy to verify that $T(t)$ is strongly continuous. The infinitesimal generator $A(C[-h, 0] \rightarrow C[-h, 0])$ is given by

$$
\begin{aligned}
\mathscr{D}(A) & =\left\{\varphi \in C[-h, 0]: \dot{\varphi} \in C[-h, 0], \dot{\varphi}(0)=\int_{0}^{h} d \zeta(\theta) \varphi(-\theta)\right\}, \\
A \varphi & =\dot{\varphi} .
\end{aligned}
$$

Lemma 1.1. Let $D(C[-h, 0] \rightarrow C[-h, 0])$ be the operator defined by

$$
\mathscr{D}(D)=\{\varphi \in C[-h, 0]: \dot{\varphi} \in C[-h, 0]\}, \quad D \varphi=\dot{\varphi} .
$$


Then $D$ satisfies the hypothesis $(\mathrm{H} 1),(\mathrm{H} 2)$ in $\S \mathrm{I} .3$ with $\Omega=\mathbb{C}$. Furthermore, the operator $A$ defined by (1.4) is the first operator associated with $D, L$ and $M$, where

$$
M \varphi=\varphi(0), \quad L \varphi=\int_{0}^{h} d \zeta(\theta) \varphi(-\theta) .
$$

Proof. Put $X=C[-h, 0]$. Clearly, the kernel of $D$ consists of the constant functions. It follows that $\mathscr{N}=\operatorname{Ker}(D)$ has dimension $n$. For $D_{0}$ we take the operator $D_{0}(X \rightarrow X)$ defined by

$$
\mathscr{D}\left(D_{0}\right)=\{\varphi \in \mathscr{D}(D): \varphi(0)=0\}, \quad D_{0} \varphi=D \varphi .
$$

We have $\mathscr{D}(D)=\mathscr{N} \oplus \mathscr{D}\left(D_{0}\right)$, and for each $z \in \mathbb{C}$ the operator $z-D_{0}$ is invertible and the resolvent of $D_{0}$ is given by

$$
\left(\left(z-D_{0}\right)^{-1} \varphi\right)(\theta)=-\int_{0}^{\theta} e^{(\theta-\sigma) z} \varphi(\sigma) d \sigma, \quad-h \leq \theta \leq 0 .
$$

Therefore $D$ satisfies $(\mathrm{H} 1),(\mathrm{H} 2)$ with $\Omega=\mathbb{C}$. Further $A$ is given by

$$
\mathscr{D}(A)=\{\varphi \in \mathscr{D}(D): M D \varphi=L \varphi\}, \quad A \varphi=D \varphi,
$$

where $L$ and $M$ are defined by (1.5). So, $A$ is the first operator associated with $D, L$ and $M$.

For the case considered here the Banach space $Z$ equals $\mathbb{C}^{n} \times C[-h, 0]$ and the second operator $\widehat{A}(Z \rightarrow Z)$ associated with the triple $D, L$ and $M$ is given by

$$
\begin{gathered}
\mathscr{D}(\widehat{A})=\left\{\left(\begin{array}{l}
c \\
\varphi
\end{array}\right) \in Z: \varphi \in \mathscr{D}(D), c=\varphi(0)\right\}, \\
\widehat{A}\left(\begin{array}{l}
c \\
\varphi
\end{array}\right)=\left(\begin{array}{c}
L \varphi \\
D \varphi
\end{array}\right) .
\end{gathered}
$$

Theorem 1.2. The matrix function $\Delta: \mathbb{C} \rightarrow \mathscr{L}\left(\mathbb{C}^{n}\right)$

$$
\Delta(z)=z I-\int_{0}^{h} e^{-z \theta} d \zeta(\theta)
$$

is a characteristic matrix for $\widehat{A}$ defined by (1.7) and the equivalence is given by

$$
\left(\begin{array}{cc}
\Delta(z) & 0 \\
0 & I
\end{array}\right)=F(z)(z-\widehat{A}) E(z), \quad z \in \mathbb{C},
$$

where $E: \mathbb{C} \rightarrow \mathscr{L}\left(Z, Z_{\widehat{A}}\right)$ is given by

$$
\begin{aligned}
E(z)\left(\begin{array}{c}
c \\
\varphi
\end{array}\right)=\left(\begin{array}{c}
c \\
\psi
\end{array}\right), \quad \psi(\theta) & =e^{\theta z} c-\int_{0}^{\theta} e^{(\theta-\sigma) z} \varphi(\sigma) d \sigma, \\
E(z)^{-1}\left(\begin{array}{c}
\psi(0) \\
\psi
\end{array}\right) & =\left(\begin{array}{c}
\psi(0) \\
(z-D) \psi
\end{array}\right),
\end{aligned}
$$

and $F: \mathbb{C} \rightarrow \mathscr{L}(Z)$ is given by

$$
\begin{gathered}
F(z)\left(\begin{array}{l}
c \\
\varphi
\end{array}\right)=\left(\begin{array}{c}
c+L\left(z-D_{0}\right)^{-1} \varphi \\
\varphi
\end{array}\right), \\
F(z)^{-1}\left(\begin{array}{l}
c \\
\varphi
\end{array}\right)=\left(\begin{array}{c}
c-L\left(z-D_{0}\right)^{-1} \varphi \\
\varphi
\end{array}\right) .
\end{gathered}
$$


Proof. Let $D$ and $D_{0}$ be as in the proof of Lemma 1.1. Define $j: \mathbb{C}^{n} \rightarrow \mathscr{N}$, $\mathscr{N}=\operatorname{Ker}(D)$, by

$$
(j c)(\theta)=c, \quad-h \leq \theta \leq 0 .
$$

From Theorem I.3.1 we know that

$$
\Delta(z)=(z M-L)\left(j-z\left(z-D_{0}\right)^{-1} j\right), \quad z \in \mathbb{C},
$$

is a characteristic matrix for $\widehat{A}$. To verify the concrete representation (1.8) we use the resolvent formula (1.6) and calculate

$$
\begin{gathered}
\left(j c-z\left(z-D_{0}\right)^{-1} j c\right)(\theta)=c+z \int_{0}^{\theta} e^{(\theta-\sigma) z} c d \sigma \\
=c-\left.e^{(\theta-\sigma) z} c\right|_{0} ^{\theta}=e^{\theta z} c, \quad-h \leq \theta \leq 0 .
\end{gathered}
$$

Finally, the concrete representations for $E$ and $F$ are verified in a similar way.

Corollary 1.3. The spectrum of $A(C[-h, 0] \rightarrow C[-h, 0])$ defined by (1.4) consists of eigenvalues of finite type only,

$$
\sigma(A)=\{\lambda: \operatorname{det} \Delta(\lambda)=0\} .
$$

For $\lambda \in \sigma(A)$, the algebraic multiplicity of the eigenvalue $\lambda$ equals the order of $\lambda$ as a zero of $\operatorname{det} \Delta$, the partial multiplicities of the eigenvalue $\lambda$ are equal to the zero-multiplicities of $\lambda$ as a characteristic value of $\Delta$, and the largest partial multiplicity (ascent) of $\lambda$ equals the order of $\lambda$ as a pole of $\Delta^{-1}$. Furthermore, a canonical basis of eigenvectors and generalized eigenvectors for $A$ at $\lambda$ may be obtained in the following way: If $\left\{\left(\gamma_{i, 0}, \ldots, \gamma_{i, k_{i}-1}\right) \mid i=1, \ldots, p\right\}$ is a canonical system of Jordan chains for $\Delta$ at $\lambda \in \Omega$, then $\left\{\chi_{i, 0}, \ldots, \chi_{i, k_{i}-1} \mid i=\right.$ $1, \ldots, p\}$, where

$$
\chi_{i, \nu}(\theta)=e^{\lambda \theta} \sum_{l=0}^{\nu} \gamma_{i, \nu-l} \frac{\theta^{l}}{l !}
$$

yields a canonical basis for $A$ at $\lambda$.

Proof. We first show that $\operatorname{det} \Delta \not \equiv 0$. From the representation (1.8) for $\Delta$ it follows that it suffices to prove

$$
\left|z^{-1} \int_{0}^{h} e^{-z t} d \zeta(t)\right| \rightarrow 0
$$

as $\Re(z) \rightarrow \infty$. But this is obvious since $\zeta$ is of bounded variation. Next we prove the representation for the canonical basis for $\hat{A}$ at $\lambda$. Let $\left(\gamma_{0}, \ldots, \gamma_{k-1}\right)$ be a Jordan chain for $\Delta$ at $\lambda$ of length $k$. Since

$$
\begin{gathered}
E(z)\left(\begin{array}{c}
\gamma_{0}+\gamma_{1}(z-\lambda)+\cdots+\gamma_{k-1}(z-\lambda)^{k-1} \\
0
\end{array}\right) \\
=\left(\begin{array}{c}
\gamma_{0}+\gamma_{1}(z-\lambda)+\cdots+\gamma_{k-1}(z-\lambda)^{k-1} \\
e_{z}\left(\gamma_{0}+\gamma_{1}(z-\lambda)+\cdots+\gamma_{k-1}(z-\lambda)^{k-1}\right)
\end{array}\right),
\end{gathered}
$$

we derive from the equivalence that

$$
e_{z}\left(\gamma_{0}+\gamma_{1}(z-\lambda)+\cdots+\gamma_{k-1}(z-\lambda)^{k-1}\right)
$$


is a root function for $(z-A)$ at $\lambda$. From the proof of part (ii) of Theorem I.2.1 it follows that we have to expand $e_{z}$ up to order $k$ in a neighbourhood of $\lambda$. Since

$$
\begin{aligned}
e_{z}(\theta) & =e^{z \theta}=e^{\lambda \theta} e^{(z-\lambda) \theta} \\
& =e^{\lambda \theta}\left[1+\theta(z-\lambda)+\cdots+\frac{\theta^{k-1}}{(k-1) !}(z-\lambda)^{k-1}+O\left((z-\lambda)^{k}\right)\right],
\end{aligned}
$$

the Jordan chain for $(z-A)$ at $\lambda$ becomes $\left(\chi_{0}, \ldots, \chi_{k-1}\right)$, where

$$
\chi_{i}=\sum_{l=0}^{i} \gamma_{i-l} \frac{\theta^{l}}{l !}(z-\lambda)^{i}
$$

and this proves the corollary.

The next corollary states the explicit form of the elementary solutions of (1.3), i.e., solutions of the form $t \mapsto p(t) e^{\lambda t}$, where $p$ is a polynomial. (Compare Hale [13, Chapter 7], for a different approach.)

Corollary 1.4. The elementary solutions of (1.3) are given by

$$
x(t)=e^{\lambda t} \sum_{l=0}^{k-1} \gamma_{(k-1)-l} \frac{t^{l}}{l !}, \quad t \geq-h,
$$

where $\left(\gamma_{0}, \ldots, \gamma_{k-1}\right)$ is a Jordan chain for $\Delta$ at $\lambda$.

Proof. From Corollary 1.3 we find $\varphi \in \mathscr{M}_{\lambda}=\operatorname{Ker}\left((\lambda I-A)^{k}\right)$ if and only if

$$
\varphi(\theta)=\sum_{l=0}^{k-1} \gamma_{(k-1)-l} \frac{\theta^{l}}{l !} e^{\lambda \theta},
$$

where $\left(\gamma_{0}, \ldots, \gamma_{k-1}\right)$ be a Jordan chain for $\Delta$ at $\lambda$. Since the solution map acts one-to-one and onto $\mathscr{M}_{\lambda}$ this shows the corollary.

Example 1.5. Consider the differential-difference equation

$$
\dot{x}(t)=x(t)-x(t-1), \quad t \geq 0,
$$

on $C[-1,0]$. The associated infinitesimal generator $A$ is given by

$$
\begin{aligned}
\mathscr{D}(A) & =\left\{\varphi \in C[-1,0]: \varphi \in C^{1}[-1,0], \dot{\varphi}(0)=\varphi(0)-\varphi(-1)\right\}, \\
A \varphi & =\dot{\varphi} .
\end{aligned}
$$

According to Theorem 1.2 the scalar function

$$
\Delta(z)=z-1+e^{-z}
$$

is a characteristic matrix for $A$. The set of zeros of $\Delta$ is infinite, but finite in any right half plane $\Re(z)>\gamma, \gamma \in \mathbb{R}$. Let us look for the finitely many zeros in the right half plane $\Re(z) \geq 0$. This yields $\lambda_{0}=0$. To find the elementary solutions of $(1.10)$ corresponding to $\lambda_{0}$, we apply the previous corollaries. The first step is to find a canonical basis of eigenvectors and generalized eigenvectors of $A$ at $\lambda_{0}=0$. To do this we first calculate the Jordan chains for $\Delta$ at $\lambda_{0}=0$. 
Since $\Delta(0)=0, \Delta^{\prime}(0)=0$, the set $\left(x_{0}, \ldots, x_{k-1}\right)$ is a Jordan chain of $\Delta$ at $\lambda_{0}$ if and only if $x_{0} \neq 0$ and

$$
\Delta(z)\left(x_{0}+x_{1} z+\cdots+x_{k-1} z^{k-1}\right)=O\left(z^{k}\right) .
$$

Hence the expression

$$
\begin{aligned}
& \left(z^{2} / 2 !-z^{3} / 3 !+\cdots\right) x_{0}+\left(z^{3} / 2 !-z^{4} / 3 !+\cdots\right) x_{1}+\cdots \\
& \quad+\left(z^{k+1} / 2 !-z^{k+2} / 3 !+\cdots\right)
\end{aligned}
$$

must be of order $O\left(z^{k}\right)$. Thus $x_{0}=x_{1}=\cdots=x_{k-3}=0$, and $x_{k-2}, x_{k-1} \in \mathbb{C}$ are arbitrary. But $x_{0}$ must be different from zero. Thus the canonical system of Jordan chains of $\Delta$ at $\lambda_{0}$ consists of one chain of length 2 . For example, we may take the chain $(1,0)$. It follows (apply Corollary 1.3$)$ that

$$
\operatorname{Ker}\left(A^{2}\right)=\operatorname{span}\left\{\varphi_{0}, \varphi_{1}\right\}
$$

where

$$
\varphi_{0}(\theta)=1, \quad \varphi_{1}(\theta)=\theta, \quad-1 \leq \theta \leq 0 .
$$

Thus the elementary solutions of (1.10) corresponding to $\lambda_{0}=0$ are $x(t)=$ $a+b t$, where $a, b \in \mathbb{C}$.

II.1.2 Product spaces. In recent years, equation (1.1) has been studied in product spaces in connection with control-theoretical applications.

Consider the initial value problem

$$
\begin{aligned}
& \dot{x}(t)=L x_{t}, \quad t \geq 0, \\
& x(0)=c, \quad x(\theta)=\varphi(\theta), \quad-h \leq \theta<0,
\end{aligned}
$$

where $c \in \mathbb{C}^{n}, \varphi \in L^{p}[-h, 0]$ and $L: L^{p}[-h, 0] \rightarrow \mathbb{C}^{n}$ are given. In Delfour [4] it was proved that the bounded linear mappings from $W^{1, p}[-h, 0]$ into $\mathbb{C}^{n}$ form the largest class of linear mappings $L$ such that $(1.11)$ is well-posed on $M_{p}=\mathbb{C}^{n} \times L^{p}[-h, 0]$. From the Riesz representation theorem any such mapping $L: W^{1, p}[-h, 0] \rightarrow \mathbb{C}^{n}$ can be written as

$$
L \varphi=\int_{0}^{h} \zeta(\theta) \varphi(-\theta) d \theta+\int_{0}^{h} \eta(\theta) \dot{\varphi}(-\theta) d \theta,
$$

where $\eta, \zeta \in L^{q}[0, h]$ and $1 / p+1 / q=1$.

So, for $L$ given by (1.12) the solution semigroup

$$
T_{p}(t)\left(\begin{array}{c}
c \\
\varphi
\end{array}\right)=\left(\begin{array}{c}
x(t ; \varphi) \\
x_{t}(\cdot ; \varphi)
\end{array}\right)
$$

is strongly continuous on $M_{p}$. Furthermore $A_{p}\left(M_{p} \rightarrow M_{p}\right)$ the infinitesimal generator is given by

$$
\begin{gathered}
\mathscr{D}\left(A_{p}\right)=\left\{\left(\begin{array}{c}
c \\
\varphi
\end{array}\right) \in M_{p}: \varphi \in W^{1, p}[-h, 0], c=\varphi(0)\right\}, \\
A_{p}\left(\begin{array}{c}
c \\
\varphi
\end{array}\right)=\left(\begin{array}{c}
L \varphi \\
\dot{\varphi}
\end{array}\right),
\end{gathered}
$$

where $L$ is given by (1.12). 
Lemma 1.6. Let $D\left(L^{p}[-h, 0] \rightarrow L^{p}[-h, 0]\right)$ be the operator defined by

$$
\mathscr{D}(D)=\left\{\varphi \in L^{p}[-h, 0]: \dot{\varphi} \in L^{p}[-h, 0]\right\}, \quad D \varphi=\dot{\varphi} .
$$

Then $D$ satisfies the hypothesis $(\mathrm{H} 1),(\mathrm{H} 2)$ in $\S 1.3$ with $\Omega=\mathbb{C}$. Furthermore, the operator $A_{p}$ defined by (1.13) is the second operator associated with $D, L$ and $M$, where $M \varphi=\varphi(0)$.

Proof. The proof of the first part follows the same argument as the proof of Lemma 1.1. The Banach space $X_{D}$ is just $W^{1, p}[-h, 0]$. For the second operator, the general construction from $\S I .3$ only requires that $L$ and $M$ are bounded linear mappings from $X_{D}$ into $\mathbb{C}^{n}$. It is given that $L$ is bounded from $W^{1, p}[-h, 0]$ into $\mathbb{C}^{n}$ and clearly the mapping $\varphi \mapsto \varphi(0)$ is a bounded linear mapping from $W^{1, p}[-h, 0]$ into $\mathbb{C}^{n}$.

Thus, we can apply Theorem I.3.1 to obtain

Theorem 1.7. The matrix function $\Delta: \mathbb{C} \rightarrow \mathscr{L}\left(\mathbb{C}^{n}\right)$

$$
\Delta(z)=z I+z \int_{0}^{h} e^{-z \theta} \eta(\theta) d z-\int_{0}^{h} e^{-z \theta} \zeta(\theta) d z
$$

is a characteristic matrix for $A_{p}\left(M_{p} \rightarrow M_{p}\right)$ defined by (1.13) and the equivalence is given by

$$
\left(\begin{array}{cc}
\Delta(z) & 0 \\
0 & I
\end{array}\right)=F(z)\left(z-A_{p}\right) E(z), \quad z \in \mathbb{C},
$$

where $E: \mathbb{C} \rightarrow \mathscr{L}\left(M_{p},\left(M_{p}\right)_{A_{p}}\right)$ is given by

$$
\begin{aligned}
E(z)\left(\begin{array}{c}
c \\
\varphi
\end{array}\right)=\left(\begin{array}{c}
c \\
\psi
\end{array}\right), \quad \psi(\theta) & =e^{\theta z} c-\int_{0}^{\theta} e^{(\theta-\sigma) z} \varphi(\sigma) d \sigma, \\
E(z)^{-1}\left(\begin{array}{c}
\psi(0) \\
\psi
\end{array}\right) & =\left(\begin{array}{c}
\psi(0) \\
(z-D) \psi
\end{array}\right)
\end{aligned}
$$

and $F: \mathbb{C} \rightarrow \mathscr{L}\left(M_{p}\right)$ is given by

$$
\begin{aligned}
F(z)\left(\begin{array}{l}
c \\
\varphi
\end{array}\right) & =\left(\begin{array}{c}
c+L\left(z-D_{0}\right)^{-1} \varphi \\
\varphi
\end{array}\right), \\
F(z)^{-1}\left(\begin{array}{l}
c \\
\varphi
\end{array}\right) & =\left(\begin{array}{c}
c-L\left(z-D_{0}\right)^{-1} \varphi \\
\varphi
\end{array}\right) .
\end{aligned}
$$

Proof. The proof is similar to the proof of Theorem 1.2. It only remains to show that $\operatorname{det} \Delta \not \equiv 0$. Since

$$
\begin{aligned}
\left\|z^{-1} L e_{z}\right\| & =\left\|\int_{0}^{h} e^{-z \theta} \eta(\theta) d \theta-z^{-1} \int_{0}^{h} e^{-z \theta} \zeta(\theta) d \theta\right\| \\
& \leq C\left(1+|z|^{-1}\right)\left[1-e^{-\Re(z) h} / \Re(z)\right] \rightarrow 0
\end{aligned}
$$

as $\Re(z) \rightarrow \infty$, it follows that $\operatorname{det} \Delta \not \equiv 0$.

Corollary 1.8. The spectrum of the operator $A_{p}\left(M_{p} \rightarrow M_{p}\right)$ defined by (1.13) consists of eigenvalues of finite type only,

$$
\sigma\left(A_{p}\right)=\{\lambda: \operatorname{det} \Delta(\lambda)=0\}
$$


and is independent of $p$. For $\lambda \in \sigma(A)$, the algebraic multiplicity of the eigenvalue $\lambda$ equals the order of $\lambda$ as a zero of $\operatorname{det} \Delta$, the partial multiplicities of the eigenvalue $\lambda$ are equal to the zero-multiplicities of $\lambda$ as a characteristic value of $\Delta$, and the largest partial multiplicity (ascent) of $\lambda$ equals the order of $\lambda$ as a pole of $\Delta^{-1}$. Furthermore, a canonical basis of eigenvectors and generalized eigenvectors for $A_{p}$ at $\lambda$ may be obtained in the following way: If $\left\{\left(\gamma_{i, 0}, \ldots, \gamma_{i, k_{i}-1}\right) \mid i=1, \ldots, p\right\}$ is a canonical system of Jordan chains for $\Delta$ at $\lambda \in \Omega$, then

where

$$
\left\{\left(\begin{array}{l}
\gamma_{i, 0} \\
\chi_{i, 0}
\end{array}\right), \ldots,\left(\begin{array}{l}
\gamma_{i, k_{i}-1} \\
\chi_{i, k_{i}-1}
\end{array}\right) \mid i=1, \ldots, p\right\}
$$

$$
\chi_{i, \nu}(\theta)=e^{\lambda \theta} \sum_{l=0}^{\nu} \gamma_{i, \nu-l} \frac{\theta^{l}}{l !}
$$

yields a canonical basis for $A_{p}$ at $\lambda$.

II.1.3 Infinite delay. For functional differential equations with a finite delay $h$, the solution operator becomes completely continuous for $t \geq h$. If the delay is infinite, the state always contains part of the initial function and the solution operator never becomes completely continuous. In this case the choice of an appropriate state is no longer trivial. For example, if $X=L^{p}(-\infty, 0]$ with $p<\infty$, then the differentiation operator $D$ has a trivial kernel and, hence the construction from $\S$ I.3 does not apply in this case. Note that for this choice of the state space the initial value problem is not well posed either.

In [14] Hale and Kato studied which properties of the state space $X$ are sufficient in order to establish a theory of existence, uniqueness, continuous dependence, and continuation of solutions. For an example of a state space that satisfies the axioms in [14], let $g$ be a nondecreasing positive function defined on $(-\infty, 0]$ such that

$$
\int_{-\infty}^{0} g(\tau) d \tau<\infty
$$

and let $p \geq 1$. Define the state space $X=L^{p}((-\infty, 0] ; g)$ to be the space of measurable functions $\varphi$ on $(-\infty, 0]$ such that, for any given $h \geq 0$, the function $\varphi$ is continuous on $[-h, 0]$ and

$$
\|\varphi\|_{g}:=\sup _{h \geq 0}\left[\sup _{-h \leq \tau \leq 0}|\varphi(\tau)|^{p}+\int_{-\infty}^{0}|\varphi(\tau)|^{p} g(\tau) d \tau\right]^{1 / p}<\infty .
$$

Thus, the initial value problem

$$
\dot{x}(t)=L x_{t}, \quad x_{0}=\varphi,
$$

where $\varphi \in L^{p}((-\infty, 0] ; g)$ and $L: L^{p}((-\infty, 0] ; g) \rightarrow \mathbb{C}^{n}$ is a bounded linear mapping, is well posed. Thus, the solution operator

$$
T(t): L^{p}((-\infty, 0] ; g) \rightarrow L^{p}((-\infty, 0] ; g)
$$

is a strongly continuous semigroup of bounded linear operators. The infinitesimal generator is given by

$$
\begin{aligned}
\mathscr{D}(A) & =\left\{\varphi \in L^{p}((-\infty, 0] ; g): \dot{\varphi} \in L^{p}((-\infty, 0] ; g), \dot{\varphi}(0)=L \varphi\right\}, \\
A \varphi & =\dot{\varphi} .
\end{aligned}
$$

(See Hale [12] and Naito [25].) 
To prove the infinite delay version of Lemma 1.1 we first need some preparations. Put

$$
\Lambda=\left\{z \in \mathbb{C}: \int_{-\infty}^{0}\left|e^{z \tau}\right|^{p} g(\tau) d \tau<\infty\right\}
$$

and define

$$
\beta=\inf \{\Re(z): z \in \Lambda\} .
$$

Then $\mathbb{C}_{\beta} \subset \Lambda \subset \overline{\mathbb{C}_{\beta}}$, where $\mathbb{C}_{\beta}=\{z \in \mathbb{C}: \Re(z)>\beta\}$ and the bar denotes the closure.

Lemma 1.9. Let $D\left(L^{p}((-\infty, 0] ; g) \rightarrow L^{p}((-\infty, 0] ; g)\right)$ be the operator defined by

$$
\mathscr{D}(D)=\left\{\varphi \in L^{p}((-\infty, 0] ; g): \dot{\varphi} \in L^{p}((-\infty, 0] ; g)\right\}, \quad D \varphi=\dot{\varphi} .
$$

Then $D$ satisfies the hypothesis $(\mathrm{H} 1),(\mathrm{H} 2)$ in $\S \mathrm{I} .3$ with $\Omega=\mathbb{C}_{\beta}$, where $\beta$ is defined by (1.18). Furthermore, the operator $A$ defined by (1.16) is the first operator associated with $D, L$ and $M$, where $M \varphi=\varphi(0)$.

Proof. Put $X=L^{p}((-\infty, 0] ; g)$. Clearly, the kernel of $D$ consists of the constant functions. It follows that $\mathscr{N}=\operatorname{Ker}(D)$ has dimension $n$. For $D_{0}$ we take the operator $D_{0}(X \rightarrow X)$ defined by

$$
\mathscr{D}\left(D_{0}\right)=\{\varphi \in \mathscr{D}(D): \varphi(0)=0\}, \quad D_{0} \varphi=D \varphi .
$$

We have $\mathscr{D}(D)=\mathscr{N} \oplus \mathscr{D}\left(D_{0}\right)$. The resolvent of $D_{0}$ is given by

$$
\left(\left(z-D_{0}\right)^{-1} \varphi\right)(\theta)=-\int_{0}^{\theta} e^{(\theta-\sigma) z} \varphi(\sigma) d \sigma, \quad z \in \rho\left(D_{0}\right) .
$$

Since the right-hand side must belong to $X$, the resolvent set of $D_{0}$ depends on $X$ and it follows that $\rho\left(D_{0}\right)=\mathbb{C}_{\beta}$, where $\beta$ is defined by (1.18). Thus $D$ satisfies $(\mathrm{H} 1)$ and $(\mathrm{H} 2)$ with $\Omega=\mathbb{C}_{\beta}$. Further the mapping $M$ given by $\varphi \mapsto \varphi(0)$ is a bounded linear mapping from $L^{p}((-\infty, 0] ; g)$ into $\mathbb{C}^{n}$ and $A$ defined by $(1.16)$ is the first operator associated with $D, L$ and $M$.

For the case considered here the Banach space

$$
Z=\mathbb{C}^{n} \times L^{p}((-\infty, 0] ; g)
$$

and the second operator $\widehat{A}(Z \rightarrow Z)$ associated with the pair $D, L$ and $M$ is given by

$$
\mathscr{D}(\widehat{A})=\left\{\left(\begin{array}{c}
c \\
\varphi
\end{array}\right) \in Z: \varphi \in \mathscr{D}(D), c=\varphi(0)\right\}, \quad \widehat{A}\left(\begin{array}{c}
c \\
\varphi
\end{array}\right)=\left(\begin{array}{c}
L \varphi \\
D \varphi
\end{array}\right) .
$$

Theorem 1.10. The matrix function $\Delta: \mathbb{C}_{\beta} \rightarrow \mathscr{L}\left(\mathbb{C}^{n}\right)$

$$
\Delta(z)=z I-L e_{z}
$$

is a characteristic matrix for $\widehat{A}$ and the equivalence is given by

$$
\left(\begin{array}{cc}
\Delta(z) & 0 \\
0 & I
\end{array}\right)=F(z)(z-\widehat{A}) E(z), \quad z \in \mathbb{C}_{\beta},
$$


where $E: \mathbb{C} \rightarrow \mathscr{L}\left(Z, Z_{\widehat{A}}\right)$ is given by

$$
\begin{aligned}
E(z)\left(\begin{array}{c}
c \\
\varphi
\end{array}\right)= & \left(\begin{array}{c}
c \\
\psi
\end{array}\right), \quad \psi(\theta)=e^{\theta z} c-\int_{0}^{\theta} e^{(\theta-\sigma) z} \varphi(\sigma) d \sigma, \\
E(z)^{-1}\left(\begin{array}{c}
\psi(0) \\
\psi
\end{array}\right) & =\left(\begin{array}{c}
\psi(0) \\
(z-D) \psi
\end{array}\right)
\end{aligned}
$$

and $F: \mathbb{C}_{\beta} \rightarrow \mathscr{L}(Z)$ is given by

$$
\begin{aligned}
F(z)\left(\begin{array}{c}
c \\
\varphi
\end{array}\right) & =\left(\begin{array}{c}
c+L\left(z-D_{0}\right)^{-1} \varphi \\
\varphi
\end{array}\right), \\
F(z)^{-1}\left(\begin{array}{c}
c \\
\varphi
\end{array}\right) & =\left(\begin{array}{c}
c-L\left(z-D_{0}\right)^{-1} \varphi \\
\varphi
\end{array}\right) .
\end{aligned}
$$

Proof. Since $e_{z} \in L^{p}((-\infty, 0] ; g)$ for $z \in \mathbb{C}_{\beta}$ the matrix function is holomorphic on $\mathbb{C}_{\beta}$ and the theorem is proved as Theorem 1.2.

Note that the equivalence is not on the whole of $\mathbb{C}$ anymore, but on $\mathbb{C}_{\beta}$. The operator $A$ has not only point spectrum. (See Naito [25].) A combination of Lemma 1.9 and Theorem 1.10 now yields the following result on the spectral data of the unbounded operator $A$ defined by (1.16).

Corollary 1.11. The eigenvalues of

$$
A\left(L^{p}((-\infty, 0] ; g) \rightarrow L^{p}((-\infty, 0] ; g)\right)
$$

defined by (1.16) with real part greater than $\beta$ (defined by (1.18)) are eigenvalues of finite type only and satisfy the equation

$$
\operatorname{det} \Delta(\lambda)=0 .
$$

For $\lambda \in \sigma(A)$ and $\Re(\lambda)>\beta$, the algebraic multiplicity of the eigenvalue $\lambda$ equals the order of $\lambda$ as a zero of $\operatorname{det} \Delta$, the partial multiplicities of the eigenvalue $\lambda$ are equal to the zero-multiplicities of $\lambda$ as a characteristic value of $\Delta$, and the largest partial multiplicity (ascent) of $\lambda$ equals the order of $\lambda$ as a pole of $\Delta^{-1}$. Furthermore, a canonical basis of eigenvectors and generalized eigenvectors for $A$ at $\lambda$ may be obtained in the following way: If $\left\{\left(\gamma_{i, 0}, \ldots, \gamma_{i, k_{i}-1}\right)\right.$ | $i=1, \ldots, p\}$ is a canonical system of Jordan chains for $\Delta$ at $\lambda \in \Omega$, then $\left\{\chi_{i, 0} \ldots, \chi_{i, k_{i}-1} \mid i=1, \ldots, p\right\}$, where

$$
\chi_{i, \nu}(\theta)=e^{\lambda \theta} \sum_{l=0}^{\nu} \gamma_{i, \nu-l} \frac{\theta^{l}}{l !},
$$

yields a canonical basis for $A$ at $\lambda$.

Proof. To prove the corollary it remains to show that $\operatorname{det} \Delta \not \equiv 0$. From the definition of the norm on $L^{p}((-\infty, 0] ; g)$ it follows that $\left\|L e_{z}\right\|_{g} \leq C|z|^{-1}$ for $\Re(z) \rightarrow \infty$, which implies that $\operatorname{det} \Delta \not \equiv 0$.

Corollary 1.12. The elementary solutions of (1.15) are given by

$$
x(t)=e^{\lambda t} \sum_{l=0}^{k-1} \gamma_{(k-1)-l} \frac{t^{l}}{l !}, \quad t \geq-h,
$$

where $\left(\gamma_{0}, \ldots, \gamma_{k-1}\right)$ is a Jordan chain for $\Delta$ at $\lambda$. 


\section{II.2. A HYPERBOLIC SYSTEM}

In this section we will study the linear autonomous hyperbolic system in one space variable in normal form. (See Lopes, Neves and Ribeiro [23].)

$$
\begin{gathered}
\frac{\partial}{\partial t}\left(\begin{array}{l}
u(t, x) \\
v(t, x)
\end{array}\right)+K(x) \frac{\partial}{\partial x}\left(\begin{array}{l}
u(t, x) \\
v(t, x)
\end{array}\right)+C(x)\left(\begin{array}{l}
u(t, x) \\
v(t, x)
\end{array}\right)=0, \\
\frac{d}{d t} M\left(\begin{array}{l}
u(t, x) \\
v(t, x)
\end{array}\right)=L\left(\begin{array}{l}
u(t, x) \\
v(t, x)
\end{array}\right)
\end{gathered}
$$

for $0 \leq x \leq l$, with boundary conditions $u(t, 0)=E v(t, 0)$, where

$$
\begin{array}{lll}
u(t, x)=\operatorname{col}\left[u_{i}(t, x)\right] & \text { for } 1 \leq i \leq N-n, & \left(\begin{array}{l}
u(t, \cdot) \\
v(t, \cdot)
\end{array}\right) \in X,
\end{array}
$$

and $M$ and $L$ are linear continuous boundary value operators

$$
M: X \rightarrow \mathbb{C}^{n}, \quad L: X \rightarrow \mathbb{C}^{n} .
$$

In addition, $E$ is an $n \times(N-n)$ matrix and $X$ is an appropriate Banach function space. Further $K$ is a $C^{1}$ invertible diagonal matrix valued function and $C$ is an arbitrary continuous matrix function. For $L$ and $M$ we can take, for example, point evaluations

$$
\begin{aligned}
& M\left(\begin{array}{l}
u(t, x) \\
v(t, x)
\end{array}\right)=v(t, l)-F u(t, l), \\
& L\left(\begin{array}{c}
u(t, x) \\
v(t, x)
\end{array}\right)=G u(t, l)+H v(t, l),
\end{aligned}
$$

where $F, G$ and $H$ are matrices of appropriate size. The system defined by (2.1) can be written as an abstract evolutionary system on $X, \dot{w}=A w$, where $A(X \rightarrow X)$ is given by

$$
\begin{aligned}
A w & =-K \frac{d w}{d x}-C w \\
\mathscr{D}(A) & =\left\{w \in X: A w \in X, u(0)=E v(0), \frac{d}{d t} M w=L w\right\} .
\end{aligned}
$$

The well-posedness problem for (2.1) can now be rephrased as the question whether $A$ generates a strongly continuous semigroup on $X$. In [23] this problem and properties of the corresponding evolutionary system have been studied in the product space frame work $X=\mathbb{C}^{n} \times L^{p}[0, l]^{N}$.

In this section we will show that the unbounded operator $A$ defined by (2.3) can be realized through the construction from $\S$ I.3. Thus, we avoid the question of $A$ generating a strongly continuous semigroup, but the equivalence we finally derive is useful in order to check the Hille-Yosida conditions.

Lemma 2.1. Let $D\left(C[0, l]^{N} \rightarrow C[0, l]^{N}\right)$ be the operator defined by

$$
\begin{aligned}
\mathscr{D}(D) & =\left\{\left(\begin{array}{l}
u \\
v
\end{array}\right) \in X:\left(\begin{array}{l}
u \\
v
\end{array}\right) \in C^{1}[0, l]^{N}, u(0)=E v(0)\right\}, \\
D\left(\begin{array}{l}
u \\
v
\end{array}\right) & =-K \frac{d}{d x}\left(\begin{array}{l}
u \\
v
\end{array}\right)-C\left(\begin{array}{l}
u \\
v
\end{array}\right) .
\end{aligned}
$$


Then $D$ satisfies the hypothesis $(\mathrm{H} 1),(\mathrm{H} 2)$ in $\S \mathrm{I} .3$ with $\Omega=\mathbb{C}$. Furthermore, the operator $A$ defined by (2.3) is the first operator associated with $D, L$ and $M$.

Proof. Put $X=C[0, l]^{N}$. To find the kernel of $D$ we solve the equation

$$
\frac{d}{d x}\left(\begin{array}{l}
u(x) \\
v(x)
\end{array}\right)=-K(x)^{-1} C(x)\left(\begin{array}{l}
u \\
v
\end{array}\right), \quad 0 \leq x \leq l .
$$

The solution is

$$
\left(\begin{array}{l}
u(x) \\
v(x)
\end{array}\right)=Y(x, 0)\left(\begin{array}{c}
u(0) \\
v(0)
\end{array}\right)=Y(x, 0)\left(\begin{array}{c}
E \\
I
\end{array}\right) v(0), \quad 0 \leq x \leq l,
$$

where

$$
Y(x, y)=e^{-\int_{y}^{x} K(s)^{-1} C(s) d s}, \quad 0 \leq y \leq x \leq l,
$$

denotes the fundamental solution with $Y(y, y)=I_{n}$. It follows that $\mathscr{N}=$ $\operatorname{Ker}(D)$ has dimension $n$. For $D_{0}$ we take the operator $D_{0}(X \rightarrow X)$ defined by

$$
\mathscr{D}\left(D_{0}\right)=\left\{\left(\begin{array}{l}
u \\
v
\end{array}\right) \in \mathscr{D}(D): v(0)=0\right\}, \quad D_{0}\left(\begin{array}{l}
u \\
v
\end{array}\right)=D\left(\begin{array}{l}
u \\
v
\end{array}\right) .
$$

Clearly, $\mathscr{D}(D)=\operatorname{Ker}(D) \oplus \mathscr{D}\left(D_{0}\right)$. To find $\Omega=\rho\left(D_{0}\right)$ we solve the equation

$$
\left(z-D_{0}\right)\left(\begin{array}{l}
u \\
v
\end{array}\right)=\left(\begin{array}{l}
f \\
g
\end{array}\right)
$$

or, equivalently

$$
\frac{d}{d x}\left(\begin{array}{l}
u \\
v
\end{array}\right)=-K^{-1}(z I+C)\left(\begin{array}{l}
u \\
v
\end{array}\right)+K^{-1}\left(\begin{array}{l}
f \\
g
\end{array}\right) .
$$

The fundamental matrix solution for the homogeneous equation

$$
\frac{d}{d x}\left(\begin{array}{l}
u \\
v
\end{array}\right)=-K^{-1}(z I+C)\left(\begin{array}{l}
u \\
v
\end{array}\right)
$$

equals

$$
\tilde{Y}(x, y, z)=e^{-z \int_{y}^{x} K(s)^{-1} d s} Y(x, y), \quad 0 \leq y \leq x \leq l,
$$

where $Y$ is given by (2.4). So, the equation (2.5) is solvable for each $z \in \mathbb{C}$. It follows that $\rho\left(D_{0}\right)=\mathbb{C}$ and the resolvent of $D_{0}$ is given by

$$
\left(\left(z-D_{0}\right)^{-1}\left(\begin{array}{l}
f \\
g
\end{array}\right)\right)(x)=\int_{0}^{x} e^{-z \int_{y}^{x} K(s)^{-1} d s} Y(x, y) K(y)^{-1}\left(\begin{array}{c}
f(y) \\
g(y)
\end{array}\right) d y
$$

for $0 \leq x \leq l$ and hence $\rho\left(D_{0}\right)=\mathbb{C}$. Thus $\Omega=\mathbb{C}$. Further the operator $A$ defined by $(2.3)$ is the first operator associated with $D, L$ and $M$, since the identity

$$
\frac{d}{d t} M\left(\begin{array}{l}
u \\
v
\end{array}\right)=M D\left(\begin{array}{l}
u \\
v
\end{array}\right)
$$

holds for the solutions of the evolutionary system

$$
\frac{d}{d t}\left(\begin{array}{l}
u \\
v
\end{array}\right)=A\left(\begin{array}{l}
u \\
v
\end{array}\right)
$$


For the case considered here the Banach space $Z$ equals $\mathbb{C}^{n} \times C[0, l]^{N}$ and the second operator $\widehat{A}(Z \rightarrow Z)$ associated with the triple $D, L$ and $M$ is given by

$$
\begin{gathered}
\mathscr{D}(\hat{A})=\left\{\left(\begin{array}{c}
c \\
w
\end{array}\right) \in Z: w \in \mathscr{D}(D), c=M w\right\}, \\
\hat{A}\left(\begin{array}{c}
c \\
w
\end{array}\right)=\left(\begin{array}{c}
L w \\
D w
\end{array}\right),
\end{gathered}
$$

where $w=\operatorname{col}[u v]$. Thus, we can apply Theorem I.3.1 to obtain

Theorem 2.2. The matrix function $\Delta: \mathbb{C} \rightarrow \mathscr{L}\left(\mathbb{C}^{n}\right)$

$$
\begin{aligned}
\Delta(z)= & z M e^{-z \int_{0}^{x} K(s)^{-1} d s} Y(x, 0)\left(\begin{array}{c}
E \\
I
\end{array}\right) \\
& -L e^{-z \int_{0}^{x} K(s)^{-1} d s} Y(x, 0)\left(\begin{array}{c}
E \\
I
\end{array}\right)
\end{aligned}
$$

is a characteristic matrix for $\hat{A}$ and the equivalence is given by

$$
\left(\begin{array}{cc}
\Delta(z) & 0 \\
0 & I
\end{array}\right)=F(z)(z-\widehat{A}) E(z),
$$

where $E: \mathbb{C} \rightarrow \mathscr{L}\left(Z, Z_{\widehat{A}}\right)$ is given by

$$
E(z)\left(\begin{array}{c}
c \\
w
\end{array}\right)=\left(\begin{array}{c}
M v_{0} \\
v_{0}
\end{array}\right)+\left(\begin{array}{c}
M v_{1} \\
v_{1}
\end{array}\right), \quad E(z)^{-1}\left(\begin{array}{c}
M v \\
v
\end{array}\right)=\left(\begin{array}{c}
v(0) \\
(z-D) v
\end{array}\right)
$$

where

$$
\begin{aligned}
& v_{0}(x)=e^{-z \int_{0}^{x} K(s)^{-1} d s} Y(x, 0)\left(\begin{array}{c}
E \\
I
\end{array}\right) c, \\
& v_{1}(x)=\int_{0}^{x} e^{-z \int_{y}^{x} K(s)^{-1} d s} Y(x, y) K(y)^{-1} w(y) d y,
\end{aligned}
$$

and $F: \mathbb{C} \rightarrow \mathscr{L}(Z)$ is given by

$$
\begin{aligned}
F(z)\left(\begin{array}{c}
c \\
w
\end{array}\right) & =\left(\begin{array}{c}
c-z M\left(z-D_{0}\right)^{-1} w+L\left(z-D_{0}\right)^{-1} w \\
w
\end{array}\right), \\
F(z)^{-1}\left(\begin{array}{c}
c \\
w
\end{array}\right) & =\left(\begin{array}{c}
c+z M\left(z-D_{0}\right)^{-1} w-L\left(z-D_{0}\right)^{-1} w \\
w
\end{array}\right) .
\end{aligned}
$$

Proof. Let $D$ and $D_{0}$ be as in the proof of Lemma 2.1. Define $j: \mathbb{C}^{n} \rightarrow \mathscr{N}$

$$
c \mapsto Y(x, 0)\left(\begin{array}{c}
E \\
I
\end{array}\right) c, \quad 0 \leq x \leq l,
$$

and put

$$
\Delta(z)=(z M-L)\left[j-z\left(z-D_{0}\right)^{-1} j\right] .
$$

From Theorem I.3.1 we know that $\Delta$ is a characteristic matrix for $\hat{A}$. To verify the concrete representation (2.9) for $\Delta$, we use the resolvent formula $(2.8)$

$$
\begin{aligned}
& \left(j c-z\left(z-D_{0}\right)^{-1} j c\right)(x) \\
& \quad=Y(x, 0)\left(\begin{array}{c}
E \\
I
\end{array}\right) c-z \int_{0}^{x} e^{-z \int_{y}^{x} K(s)^{-1} d s} K(y)^{-1} d y Y(x, 0)\left(\begin{array}{c}
E \\
I
\end{array}\right) c \\
& =e^{-z \int_{0}^{x} K(s)^{-1} d s} Y(x, 0)\left(\begin{array}{c}
E \\
I
\end{array}\right) c, \quad 0 \leq x \leq l,
\end{aligned}
$$


where we use that $K$ is a diagonal matrix. The explicit representations for $E$ and $F$ are verified in a similar way.

A combination of Lemma 2.1 and Theorem 2.2 now yields the following result on the spectral data of the unbounded operator $A$ defined by (2.3).

Corollary 2.3. If $\operatorname{det} \Delta \not \equiv 0$, then the spectrum of $A\left(C[0, l]^{N} \rightarrow C[0, l]^{N}\right) d e$ fined by (2.3) consists of eigenvalues of finite type only,

$$
\sigma(A)=\{\lambda: \operatorname{det} \Delta(\lambda)=0\} .
$$

For $\lambda \in \sigma(A)$, the algebraic multiplicity of the eigenvalue $\lambda$ equals the order of $\lambda$ as a zero of $\operatorname{det} \Delta$, the partial multiplicities of the eigenvalue $\lambda$ are equal to the zero-multiplicities of $\lambda$ as a characteristic value of $\Delta$, and the largest partial multiplicity (ascent) of $\lambda$ equals the order of $\lambda$ as a pole of $\Delta^{-1}$. Furthermore, a canonical basis of eigenvectors and generalized eigenvectors for $A$ at $\lambda$ may be obtained in the following way: If $\left\{\left(\gamma_{i, 0}, \ldots, \gamma_{i, k_{i}-1}\right) \mid i=1, \ldots, p\right\}$ is a canonical system of Jordan chains for $\Delta$ at $\lambda \in \Omega$, then

$$
\left\{\left(\begin{array}{c}
E \chi_{i, 0} \\
\chi_{i, 0}
\end{array}\right), \ldots,\left(\begin{array}{c}
E \chi_{i, k_{i}-1} \\
\chi_{i, k_{i}-1}
\end{array}\right) \mid i=1, \ldots, p\right\},
$$

where

$$
\chi_{i, \nu}(t)=e^{-\lambda \int_{0}^{x} K(s)^{-1} d s} \sum_{l=0}^{\nu} \frac{\left[-\int_{0}^{x} K(s)^{-1} d s\right]^{l}}{l !} Y(x, 0) \gamma_{i, \nu-l}
$$

yields a canonical basis for $A$ at $\lambda$.

\section{II.3. NEUTRAL FUNCTIONAL DIFFERENTIAL EQUATIONS}

For retarded functional differential equations, equation (1.1) yields a bound for the derivative of the solution. Thus retarded equations are smoothing. In particular, the solution map becomes completely continuous for $t \geq h$.

In neutral functional differential equations, there may appear derivatives with a delay and those equations are in general not smoothing.

Consider a neutral functional differential equation (NFDE). The general form of the equation is as follows

$$
\frac{d}{d t} M x_{t}=L x_{t} \text { for } t \geq 0,
$$

where $L, M: X \rightarrow \mathbb{R}^{n}$ are bounded linear mappings. (See Hale [13] and Henry [15].)

As before the state space $X$ equals $C[-h, 0]$ and consequently, the "functionals" $L$ and $M$ can be represented in a unique way as Riemann-Stieltjes integrals

$$
\begin{aligned}
L \varphi & =\int_{0}^{h} d \zeta(\theta) \varphi(-\theta), \\
M \varphi & =\int_{0}^{h} d \eta(\theta) \varphi(-\theta),
\end{aligned}
$$

where $\zeta$ and $\eta$ are $n \times n$-matrices of bounded variation on $[0, h]$. Further the state $x_{t}$ is given by

$$
x_{t}(\theta)=x(t+\theta) \text { for }-h \leq \theta \leq 0 .
$$


Only under additional assumptions on $\eta$ the problem becomes well posed. The minimal assumptions are not clear. (See Burns, Herdman, Stech [2].) It is sufficient to assume that $\eta$ has an atom at 0 , that is,

$$
M \varphi=\varphi(0)-\int_{0}^{h} d \mu(\theta) \varphi(-\theta)
$$

where $\mu$ is continuous at zero. The abstract Cauchy problem associated with (3.1) is given by $\dot{u}=A u$, where $A(C[-h, 0] \rightarrow C[-h, 0])$ is given by

$$
\mathscr{D}(A)=\left\{\varphi \in C[-h, 0]: \varphi \in C^{1}[-h, 0], M \dot{\varphi}=L \varphi\right\}, \quad A \varphi=\dot{\varphi} .
$$

Again, we shall only study $A$ as an unbounded operator and our results hold independently from $A$ being a generator of a strongly continuous semigroup on $X$. Thus, we can assume that $M$ satisfies (3.3). The following lemma and theorem are similar to Lemma 1.1 and Theorem 1.2 and the proofs will be omitted.

Lemma 3.1. Let $D(C[-h, 0] \rightarrow C[-h, 0])$ be the operator defined by

$$
\mathscr{D}(D)=\{\varphi \in C[-h, 0]: \dot{\varphi} \in C[-h, 0]\}, \quad D \varphi=\dot{\varphi} .
$$

Then $D$ satisfies the hypothesis $(\mathrm{H} 1),(\mathrm{H} 2)$ in $\S \mathrm{I} .3$ with $\Omega=\mathbb{C}$. Furthermore, the operator $A$ defined by (3.5) is the first operator associated with $D, L$ and $M$, where $L$ is defined by (3.2) and $M$ by (3.3).

For the case considered here the Banach space $Z$ equals $\mathbb{C}^{n} \times C[-h, 0]$ and the second operator $\widehat{A}(Z \rightarrow Z)$ associated with the triple $D, L$ and $M$ is given by

$$
\mathscr{D}(\widehat{A})=\left\{\left(\begin{array}{c}
c \\
\varphi
\end{array}\right) \in Z: \varphi \in \mathscr{D}(D), c=M \varphi\right\}, \quad \widehat{A}\left(\begin{array}{c}
c \\
\varphi
\end{array}\right)=\left(\begin{array}{c}
L \varphi \\
D \varphi
\end{array}\right)
$$

and $M$ is defined by (3.3). Thus, we can apply Theorem I.3.1 to obtain

Theorem 3.2. The matrix function $\Delta: \mathbb{C} \rightarrow \mathscr{L}\left(\mathbb{C}^{n}\right)$

$$
\Delta(z)=z \int_{0}^{h} e^{-z \theta} d \eta(\theta)-\int_{0}^{h} e^{-z \theta} d \zeta(\theta)
$$

is a characteristic matrix for $\widehat{A}$ and the equivalence is given by

$$
\left(\begin{array}{cc}
\Delta(z) & 0 \\
0 & I
\end{array}\right)=F(z)\left(z-\widehat{A_{j}} E(z)\right.
$$

where $E: \mathbb{C} \rightarrow \mathscr{L}\left(Z, Z_{\widehat{A}}\right)$ is given by

$$
\begin{gathered}
E(z)\left(\begin{array}{c}
c \\
\varphi
\end{array}\right)=\left(\begin{array}{c}
M \psi \\
\psi
\end{array}\right), \quad \psi(\theta)=e^{\theta z} c-\int_{0}^{\theta} e^{(\theta-\sigma) z} \varphi(\sigma) d \sigma \\
E(z)^{-1}\left(\begin{array}{c}
M \psi \\
\psi
\end{array}\right)=\left(\begin{array}{c}
\psi(0) \\
(z-D) \psi
\end{array}\right)
\end{gathered}
$$

and $F: \mathbb{C} \rightarrow \mathscr{L}(Z)$ is given by

$$
\begin{aligned}
F(z)\left(\begin{array}{l}
c \\
\varphi
\end{array}\right) & =\left(\begin{array}{c}
c-z M\left(z-D_{0}\right)^{-1} \varphi+L\left(z-D_{0}\right)^{-1} \varphi \\
\varphi
\end{array}\right), \\
F(z)^{-1}\left(\begin{array}{l}
c \\
\varphi
\end{array}\right) & =\left(\begin{array}{c}
c+z M\left(z-D_{0}\right)^{-1} \varphi-L\left(z-D_{0}\right)^{-1} \varphi \\
\varphi
\end{array}\right) .
\end{aligned}
$$


Corollary 3.3. Suppose that $M$ is atomic and given by (3.4), then the spectrum of the operator $A(C[-h, 0] \rightarrow C[-h, 0])$ defined by (3.5) consists of eigenvalues of finite type only,

$$
\sigma(A)=\{\lambda: \operatorname{det} \Delta(\lambda)=0\}
$$

For $\lambda \in \sigma(A)$, the algebraic multiplicity of the eigenvalue $\lambda$ equals the order of $\lambda$ as a zero of $\operatorname{det} \Delta$, the partial multiplicities of the eigenvalue $\lambda$ are equal to the zero-multiplicities of $\lambda$ as a characteristic value of $\Delta$, and the largest partial multiplicity (ascent) of $\lambda$ equals the order of $\lambda$ as a pole of $\Delta^{-1}$. Furthermore, a canonical basis of eigenvectors and generalized eigenvectors for $A$ at $\lambda$ may be obtained in the following way: If $\left\{\left(\gamma_{i, 0}, \ldots, \gamma_{i, k_{i}-1}\right) \mid i=1, \ldots, p\right\}$ is $a$ canonical system of Jordan chains for $\Delta$ at $\lambda \in \Omega$, then $\left\{\chi_{i, 0}, \ldots, \chi_{i, k_{i}-1} \mid i=\right.$ $1, \ldots, p\}$, where

$$
\chi_{i, \nu}(\theta)=e^{\lambda \theta} \sum_{l=0}^{\nu} \gamma_{i, \nu-l} \frac{\theta^{l}}{l !}
$$

yields a canonical basis for $A$ at $\lambda$.

Proof. To prove the corollary it remains to show that $\operatorname{det} \Delta \not \equiv 0$. From the representation (3.6) for $\Delta$ it follows that

$$
\operatorname{det} \Delta(z)=z^{n}\left[1-\operatorname{det} \int_{0}^{h} e^{-z t} d \mu(t)\right]+\sum_{j=1}^{n} \int_{0}^{j h} e^{-z t} d \nu_{j}(t) z^{n-j}
$$

where $\nu_{j}$ for $1 \leq j \leq n$ are functions of bounded variations. Since $\mu$ is continuous at zero, there is a constant $C$, such that, $|\operatorname{det} \Delta(z)| \geq C|z|^{n}$ for $\Re(z)$ sufficiently large.

Remark 3.4. For the present case, the spectral situation is different from the retarded case. In general, equation (3.8) can have infinitely many zeros in a vertical strip.

\section{II.4. AGE-DEPENDENT POPUlATION DYNAMICAL MODELS}

In this last example we consider a model from the age-dependent population dynamics. (See Webb [31].) The model is formulated as a conservation law and the variable $p$ denotes the population density with respect to age $a$ at time $t$. The time evolution is governed by the Lotka-McKendrick-von Förster equation

$$
\begin{aligned}
\left(\frac{\partial}{\partial a}+\frac{\partial}{\partial t}\right) p(a, t) & =-\mu(a) p(a, t), \quad t>0,0<a<\omega \\
p(0, t) & =\int_{0}^{\omega} \beta(a) p(a, t) d a, \quad t>0 \\
p(a, 0) & =\varphi(a), \quad 0 \leq a \leq \omega,
\end{aligned}
$$

where $\varphi \in X=L^{1}[0, \omega]$ and $\mu, \beta \in L^{\infty}[0, \omega]$. Here $\mu$ represents the mortality modulus, i.e., $\exp \left(-\int_{a_{1}}^{a_{2}} \mu(a) d a\right)$ is the probability of survival from $a_{1}$ to $a_{2}$ and $\beta$ denotes the birth modulus. 
The abstract evolutionary system for (4.1) is given by $\dot{u}=A u$, where $A\left(L^{1}[0, \omega] \rightarrow L^{1}[0, \omega]\right)$ is defined by

$$
\begin{gathered}
\mathscr{D}(A)=\left\{\varphi \in L^{1}[0, \omega]: \varphi \in W^{1,1}[0, \omega], \varphi(0)=\int_{0}^{\omega} \beta(a) \varphi(a) d a\right\}, \\
A \varphi=-\frac{d \varphi}{d a}-\mu \varphi
\end{gathered}
$$

where $W^{1,1}[0, \omega]$ can be identified with the space of absolutely continuous functions on $[0, \omega]$ and $[0, \omega]=[0, \infty)$ if $\omega=\infty$. Using the method of characteristics (see Webb [31]), we can solve the problem and $A$ generates a strongly continuous semigroup of linear operators on $L^{1}[0, \omega]$ given by

$$
(T(t) \varphi)(a)=p(t, a) .
$$

Lemma 4.1. Let $D\left(L^{1}[0, \omega] \rightarrow L^{1}[0, \omega]\right)$ be the operator defined by

$$
\mathscr{D}(D)=\left\{\varphi \in L^{1}[0, \omega]: \varphi \in A C[0, \omega]\right\}, \quad D \varphi=-\frac{d \varphi}{d a}-\mu \varphi .
$$

Then $D$ satisfies the hypothesis $(\mathrm{H} 1),(\mathrm{H} 2)$ in $\S \mathrm{I} .3$ with $\Omega=\rho\left(D_{0}\right) \neq \varnothing$. Furthermore, the operator $A$ defined by (4.2) is the first operator associated with $D, L$ and $M$, where

$$
M \dot{\varphi}=0, \quad L \varphi=\varphi(0)-\int_{0}^{\omega} \beta(a) \varphi(a) d a .
$$

Proof. Put $X=L^{1}[0, \omega]$. To find the kernel of $D$ we solve the equation

$$
\frac{d \varphi}{d a}(a)=-\mu(a) \varphi(a), \quad 0 \leq a \leq \omega .
$$

The solution is

$$
\varphi(a)=Y(a, 0) \varphi(0), \quad 0 \leq a \leq \omega
$$

where

$$
Y(a, y)=e^{-\int_{y}^{a} \mu(s) d s}, \quad 0 \leq y \leq a \leq \omega,
$$

denotes the fundamental matrix solution of (4.4). It follows that $\mathscr{N}=\operatorname{Ker}(D)$ has dimension $n$. For $D_{0}$ we take the operator $D_{0}(X \rightarrow X)$ defined by

$$
\mathscr{D}\left(D_{0}\right)=\{\varphi \in \mathscr{D}(D): \varphi(0)=0\}, \quad D_{0} \varphi=D \varphi .
$$

Clearly, $\mathscr{D}(D)=\operatorname{Ker}(D) \oplus \mathscr{D}\left(D_{0}\right)$. To find $\Omega=\rho\left(D_{0}\right)$ we solve the equation $\left(z-D_{0}\right) \varphi=\psi$ or, equivalently

$$
\frac{d \varphi}{d a}=-(z+\mu) \varphi+\psi
$$

The fundamental matrix solution for the homogeneous equation is equal to $e^{-z a} Y(a, y)$, where $Y(a, y)$ is given by (4.4). Thus, the resolvent of $D_{0}$ is given by

$$
\left(\left(z-D_{0}\right)^{-1} \psi\right)(a)=\int_{0}^{a} e^{-z(a-y)} Y(a, y) \psi(y) d y, \quad 0 \leq a \leq \omega .
$$


If $\omega<\infty$, then the equation (4.6) is solvable for each $z \in \mathbb{C}$ and $\rho\left(D_{0}\right)=\mathbb{C}$. If $\omega=\infty$, then $\rho\left(D_{0}\right) \neq \mathbb{C}$. But $\rho\left(D_{0}\right) \neq \varnothing$. In particular, if $\mu_{1} \leq \mu \leq \mu_{2}$ in the sense that

$$
-\mu_{2}|x| \leq \sum_{j=1}^{n} \operatorname{sign} x_{j}(-\mu(a) x)_{j} \leq-\mu_{1}|x|
$$

for all $x \in \mathbb{C}^{n}$ and $a \geq 0$, then it is clear that

$$
\mathbb{C}_{\mu_{1}} \subset \rho\left(D_{0}\right) \subset \mathbb{C}_{\mu_{2}} .
$$

Thus $D$ satisfies the hypotheses $(\mathrm{H} 1),(\mathrm{H} 2)$. Finally, let $M$ and $L$ be as in (4.3). Note that $L$ is a bounded mapping from the domain of $D$-the absolutely continuous functions-into $\mathbb{C}^{n}$ and with this choice of $M$ and $L$ we find that $A$ equals the first operator associated with $D, L$ and $M$.

For the case considered here the Banach space $Z$ equals $\mathbb{C}^{n} \times C[0, \omega]$ and the second operator $\widehat{A}(Z \rightarrow Z)$ associated with the triple $D, L$ and $M$ is given by

$$
\begin{gathered}
\mathscr{D}(\widehat{A})=\left\{\left(\begin{array}{c}
c \\
w
\end{array}\right) \in Z: w \in \mathscr{D}(D), c=0\right\}, \\
\widehat{A}\left(\begin{array}{c}
c \\
w
\end{array}\right)=\left(\begin{array}{c}
L w \\
D w
\end{array}\right) .
\end{gathered}
$$

Theorem 4.2. The matrix operator $\Delta: \Omega \rightarrow \mathscr{L}\left(\mathbb{C}^{n}\right)$

$$
\Delta(z)=I-\int_{0}^{\omega} e^{-z a} \beta(a) e^{\int_{0}^{a} \mu(s) d s} d a .
$$

is a characteristic matrix for $\widehat{A}$ defined by (4.9) and the equivalence is given by

$$
\left(\begin{array}{cc}
\Delta(z) & 0 \\
0 & I
\end{array}\right)=F(z)(z-\widehat{A}) E(z),
$$

where $E: \mathbb{C} \rightarrow \mathscr{L}\left(Z, Z_{\widehat{A}}\right)$ is given by

$$
E(z)\left(\begin{array}{c}
c \\
\varphi
\end{array}\right)=\left(\begin{array}{c}
0 \\
\psi_{1}
\end{array}\right), \quad E(z)^{-1}\left(\begin{array}{c}
0 \\
\psi
\end{array}\right)=\left(\begin{array}{c}
\psi(0) \\
(z-D) \psi
\end{array}\right),
$$

where

$$
\psi_{1}(a)=e^{-z a}\left[e^{-\int_{0}^{a} \mu(s) d s} c+\int_{0}^{a} e^{-z \theta} e^{-\int_{0}^{\theta} \mu(s) d s} \varphi(\theta) d \theta\right]
$$

and $F: \mathbb{C} \rightarrow \mathscr{L}(Z)$ is given by

$$
\begin{aligned}
F(z)\left(\begin{array}{l}
c \\
\varphi
\end{array}\right) & =\left(\begin{array}{c}
c+L\left(z-D_{0}\right)^{-1} \varphi \\
\varphi
\end{array}\right), \\
F(z)^{-1}\left(\begin{array}{l}
c \\
\varphi
\end{array}\right) & =\left(\begin{array}{c}
c-L\left(z-D_{0}\right)^{-1} \varphi \\
\varphi
\end{array}\right) .
\end{aligned}
$$

Proof. Let $D$ and $D_{0}$ be as in the proof of Lemma 2.1. Define $j: \mathbb{C}^{n} \rightarrow \mathcal{N}$ by

$$
c \mapsto Y(a, 0) c,
$$


and put

$$
\Delta(z)=(z M-L)\left(j-z\left(z-D_{0}\right)^{-1} j\right) .
$$

From Theorem I.3.1 we know that $\Delta$ is a characteristic matrix function for $\widehat{A}$. To verify the concrete representation $(4.10)$ for $\Delta$, we use the resolvent formula (4.7)

$$
\begin{aligned}
\left(j c-z\left(z-D_{0}\right)^{-1} j c\right)(a) & =Y(a, 0) c-z \int_{0}^{a} e^{-z(a-y)} d y Y(a, 0) c \\
& =e^{-z a} Y(a, 0) c, \quad 0 \leq a \leq \omega .
\end{aligned}
$$

The concrete representations for $E$ and $F$ are verified in a similar manner.

Thus, a combination of Lemma 4.1 and Theorem 4.2 yields the following results on the spectral data of the unbounded operator $A$ defined by (4.2).

Corollary 4.3. If $\omega<\infty$, then the spectrum of the operator

$$
A\left(L^{1}[0, \omega] \rightarrow L^{1}[0, \omega]\right)
$$

defined by (4.2) consists of eigenvalues of finite type only,

$$
\sigma(A)=\{\lambda: \operatorname{det} \Delta(\lambda)=0\} .
$$

For $\lambda \in \sigma(A)$, the algebraic multiplicity of the eigenvalue $\lambda$ equals the order of $\lambda$ as a zero of $\operatorname{det} \Delta$, the partial multiplicities of the eigenvalue $\lambda$ are equal to the zero-multiplicities of $\lambda$ as a characteristic value of $\Delta$, and the largest partial multiplicity (ascent) of $\lambda$ equals the order of $\lambda$ as a pole of $\Delta^{-1}$. Furthermore, a canonical basis of eigenvectors and generalized eigenvectors for $A$ at $\lambda$ may be obtained in the following way: If $\left\{\left(\gamma_{i, 0}, \ldots, \gamma_{i, k_{i}-1}\right) \mid i=1, \ldots, p\right\}$ is $a$ canonical system of Jordan chains for $\Delta$ at $\lambda \in \Omega$, then $\left\{\chi_{i, 0} \ldots, \chi_{i, k_{i}-1} \mid i=\right.$. $1, \ldots, p\}$, where

$$
\chi_{i, \nu}(x)=e^{\lambda x} \sum_{l=0}^{\nu} Y(x, a) \gamma_{i, \nu-l} \frac{x^{l}}{l !},
$$

yields a canonical basis for $A$ at $\lambda$.

Proof. To prove the corollary it remains to show that $\operatorname{det} \Delta \not \equiv 0$. From the representation (4.9) for $\Delta$ it follows that

$$
\operatorname{det} \Delta(z)=I+\sum_{l=1}^{n} \int_{0}^{l \omega} e^{-z t} \beta_{l}(t) d t,
$$

where $\beta_{l}$ for $1 \leq l \leq n$ are integrable functions. In particular, this shows that $\operatorname{det} \Delta$ is not identically zero.

In case $\omega=\infty$ we have the following information about the spectral data of $A$ defined by (4.2). (See Desch and Schappacher [6].)

Corollary 4.4. If $\omega=\infty$, then the eigenvalues of

$$
A\left(L^{1}[0, \infty) \rightarrow L^{1}[0, \infty)\right)
$$

defined by (4.2) with real part greater than $\mu_{1}$ (defined by (4.8)) are eigenvalues of finite type and roots of the equation

$$
\operatorname{det} \Delta(\lambda)=0
$$


For $\lambda \in \sigma(A)$ and $\Re(\lambda)>\mu_{1}$, the algebraic multiplicity of the eigenvalue $\lambda$ equals the order of $\lambda$ as a zero of $\operatorname{det} \Delta$, the partial multiplicities of the eigenvalue $\lambda$ are equal to the zero-multiplicities of $\lambda$ as a characteristic value of $\Delta$, and the largest partial multiplicity (ascent) of $\lambda$ equals the order of $\lambda$ as a pole of $\Delta^{-1}$. Furthermore, a canonical basis of eigenvectors and generalized eigenvectors for $A$ at $\lambda$ may be obtained in the following way: If $\left\{\left(\gamma_{i, 0}, \ldots, \gamma_{i, k_{i}-1}\right) \mid i=1, \ldots, p\right\}$ is a canonical system of Jordan chains for $\Delta$ at $\lambda \in \Omega$, then $\left\{\chi_{i, 0} \ldots, \chi_{i, k_{i}-1} \mid i=1, \ldots, p\right\}$, where

$$
\chi_{i, \nu}(x)=e^{\lambda x} \sum_{l=0}^{\nu} Y(x, a) \gamma_{i, \nu-l} \frac{x^{l}}{l !},
$$

yields a canonical basis for $A$ at $\lambda$.

Proof. The property that $\operatorname{det} \Delta \not \equiv 0$ does not depend upon $\omega$. Thus the corollary follows.

\section{REFERENCES}

1. H. Bart, I. Gohberg and M.A. Kaashoek, Minimal factorization of matrix and operator functions, Birkhäuser Verlag, Basel, 1979.

2. J. A. Burns, T. L. Herdman and H. W. Stech, Linear functional differential equations as semigroups on product spaces, SIAM J. Math. Anal. 14 (1983), 98-116.

3. Ph. Clément, O. Diekmann, M. Gyllenberg, H. J. A. M. Heijmans, and H. R. Thieme, Perturbation theory for dual semigroups; The sun-reflexive case, Math. Ann. 277 (1987), 709-725.

4. M. Delfour, The largest class of hereditary systems defining a $\mathscr{C}_{0}$-semigroup, Canad. J. Math. 32 (1980), 969-975.

5. O. Diekmann and S. M. Verduyn Lunel, A new short proof of an old folk theorem in functional differential equations, Semigroup Theory and Evolution Equations (Philippe Clément, Enzo Mitidieri and Ben de Pagter, ed.), Lecture Notes in Pure and Applied Mathematics, Vol. 135, Marcel Dekker, New York, pp. 101-107.

6. W. Desch and W. Schappacher, Spectral properties of finite-dimensional perturbed linear semigroups, J. Differential Equations 59 (1985), 80-102.

7. N. Dunford and J. T. Schwartz, Linear operators, Part I: General theory, Wiley, New York, 1958.

8. F. R. Gantmacher, Matrizenrechnung, Teil I, VEB Deutsche Verlag der Wissenschaften, Berlin.

9. I. Gohberg, M. A. Kaashoek and D. C. Lay, Equivalence, linearization, and decomposition of holomorphic operator functions, J. Funct. Anal. 28 (1978), 102-144.

10. I. Gohberg, S. Goldberg and M. A. Kaashoek, Classes of linear operators I, Birkhäuser Verlag, Basel, 1990.

11. I. C. Gohberg and E. I. Sigal, An operator generalization of the logarithmic residue theorem and the theorem of Rouché, Mat. Sb. 84 (1971), 609-629; English transl., Math. USSR-Sb. 13 (1971), 603-625.

12. J. K. Hale, Functional differential equations with infinite delay, J. Math. Anal. Appl. 48 (1974), 276-283.

13. __ Theory of functional differential equations, Springer-Verlag, Berlin, 1977.

14. J. K. Hale and J. Kato, Phase space for retarded equations with infinite delay, Funkcial. Ekvac. 21 (1978), 11-41.

15. D. Henry, Linear autonomous neutral functional differential equations, J. Differential Equations 15 (1974), 106-128. 
16. E. Hille and R. Phillips, Functional analysis and semigroups, Amer. Math. Soc., Providence, R.I., 1957.

17. N. Jacobson, Lectures in abstract algebra, Volume II, Springer-Verlag, Berlin, 1975.

18. M. A. Kaashoek, Analytic equivalence of the boundary eigenvalue operator function and its characteristic matrix function, Integral Equations and Operator Theory 9 (1986), 275-285.

19. F. Kappel and H. K. Wimmer, An elementary divisor theory for autonomous linear functional differential equations, J. Differential Equations 21 (1976), 346-478.

20. T. Kato, Perturbation theory for linear operators, (2nd ed.), Springer-Verlag, Berlin, 1976.

21. H. Langer and M. Möller, Linearization of boundary eigenvalue problems, Integral Equations and Operator Theory (to appear).

22. B. W. Levinger, A folk theorem in functional differential equations, J. Differential Equations 4 (1968), 612-619.

23. O. F. Lopes, A. F. Neves and H. S. Ribeiro, On the spectrum of evolution operators generated by hyperbolic systems, J. Funct. Anal. 67 (1986), 320-344.

24. R. Mennicken and M. Möller, Root functions of boundary eigenvalue operator functions, Integral Equations and Operator Theory 9 (1986), 237-265.

25. T. Naito, On autonomous linear functional differential equations with infinite retardations, J. Differential Equations 21 (1976), 297-315.

26. A. F. Neves and X.-B. Lin, A multiplicity theorem for hyperbolic systems, J. Differential Equations 75 (1988), 339-352.

27. A. Pazy, Semigroups of linear operators and applications to partial differential equations, Springer-Verlag, Berlin, 1983.

28. P. J. Rabier, Generalized Jordan chains and two bifurcation theorems of Krasnoselskii, Nonlinear Anal., Theory Methods and Appl. 13 (1989), 903-934.

29. A. E. Taylor and D. C. Lay, Introduction to functional analysis, Wiley, New York, 1980.

30. S. M. Verduyn Lunel, Exponential type calculus for linear delay equations, Tract No. 57, Centre for Mathematics and Computer Science, Amsterdam, 1989.

31. G. Webb, Theory of nonlinear age-dependent population dynamics, Marcel Dekker, New York, 1985.

Faculteit Wiskunde en Informatica, Vrije Universiteit, de BoelelaAn 1081A, $1081 \mathrm{HV}$ AMSTERdaM, The Netherlands

School of Mathematics, Georgia Institute of Technology, atlanta, Georgia 30332 